Research Article

\title{
Analysis of Memreactance with Fractional Kinetics
}

\author{
Rawid Banchuin (10) \\ Faculty of Engineering and Graduated School of Information Technology, Siam University, Bangkok, Thailand \\ Correspondence should be addressed to Rawid Banchuin; rawid_b@yahoo.com
}

Received 1 November 2019; Accepted 31 December 2019; Published 21 January 2020

Academic Editor: Pietro Bia

Copyright (c) 2020 Rawid Banchuin. This is an open access article distributed under the Creative Commons Attribution License, which permits unrestricted use, distribution, and reproduction in any medium, provided the original work is properly cited.

In this work, the analysis of the memreactance, i.e., meminductor and memcapacitor, with fractional-order kinetics has been proposed. The meminductances, memcapacitances, and related parameters due to both DC and periodic input waveforms have been derived. The behavioral analysis has been thoroughly performed with the aid of numerical simulation. The effects of fractional-order kinetics have been explored where both linear and nonlinear dopant drift scenarios have been considered. Moreover, the emulation of memreactance with fractional-order kinetics by using the memristor and the effect of the fractionalorder kinetics on the memreactance-based circuits have also been mentioned along with the extension of our results to the fractional-order memreactance.

\section{Introduction}

Apart from the basic circuit elements, the circuit elements with memory, i.e., memristor, meminductor, and memcapacitor, have been found by Leon Chua and his colleagues $[1,2]$. The kinetics of the memristor has been further generalized in the fractional-order domain as proposed in previous works [3-7] by using the concept of fractional calculus. By such a concept, the fractional derivative which is capable of including the effect of the past state, i.e., memory effect, of any system of the interested unlike the conventional derivative, has been used in the mathematical analysis. There exist many fractional derivatives, e.g., Riemann-Liouville, Liouville-Caputo [8], Caputo-Fabrizio [9], and Atangana-Baleanu [10]. Some of them, e.g., Riemann-Liouville and Liouville-Caputo derivatives, relied on the simple powerlaw kernel, whereas the others, e.g., Caputo-Fabrizio and Atangana-Baleanu fractional derivatives, employ more complicated kernel functions, e.g., exponential and Mittag-Leffler functions. As a result, different fractional derivatives describe the effect of the past state, i.e., memory effect, of an arbitrary system in different manners. The applications of the fractional calculus concept and fractional derivatives can be found in many research areas, e.g., biomedical engineering $[11,12]$, control system [13-15], electrical/electronic engineering [16-30], and plasma physics [31, 32].

Motivated by the generalization of memristor kinetics, we generalize the kinetics of the meminductor and memcapacitor which are commonly referred to as memreactance [33-35] and have been adopted in many applications, e.g., electronic oscillator [36] and synaptic circuit $[37,38]$, in the fractional-order domain by applying the fractional calculus concept to the state equation of the memreactance and perform the modelling of such memreactance. By using the obtained results, the meminductances, memcapacitances, and related parameters due to both DC and periodic input waveforms have been derived. Moreover, the behavioral analysis has been thoroughly performed with the aid of numerical simulation with MATHEMATICA. The effects of fractional-order kinetics have been explored where both linear and nonlinear dopant drift scenarios have been considered. The emulation of memreactance with fractional-order kinetics by using the memristor and the effect of the fractionalorder kinetics on the memreactance-based circuits have also been mentioned. In addition, the extension of our results to the fractional-order memreactance which employs the interpolate characteristics between the memristor and the memreactance has also been presented. 
In the following section, the overview of both the meminductor and the memcapacitor will be briefly given followed by the proposed fractional domain generalization and mathematical model in Section 3. The resulting meminductances, memcapacitances, and related parameters due to various input waveforms and the behavioral analysis will be given in Section 4 where the DC waveform will be firstly treated followed by the periodic ones. The emulation by using the memristor will be discussed in Section 5. Moreover, the effect of the fractional-order kinetics on the memreactance-based circuits will be studied in Section 6, and the extension of our results to the fractional-order memreactance will be shown in Section 7. Finally, the conclusion will be drawn in Section 8.

\section{The Overview of Meminductor and Memcapacitor}

Meminductor and memcapacitor are nonlinear electrical circuit elements. They can be simply thought of as the inductor and capacitor with memory. The meminductor relates the time integral of flux $(\rho(t))$ and instantaneous charge $(q(t))$ through the following constitutive relation [5]:

$$
L_{M}(t)=\frac{\mathrm{d} \rho(t)}{\mathrm{d} q(t)},
$$

where $L_{M}(t)$ denotes the memductance. Since we assume the electromechanical model of the meminductor and the current controlled operation [39] in this work, $L_{M}(t)$ can be given in terms of its minimum and maximum values denoted by $L_{\min }$ and $L_{\max }$ and the state variable $\left(x_{L}(t)\right)$ as

$$
\sqrt{L_{M}(t)}=\sqrt{L_{\min }}+x_{L}(t)\left(\sqrt{L_{\max }}-\sqrt{L_{\min }}\right)
$$

where $x_{L}(t)$ can be given in terms of the meminductor's current $(i(t))$ and mobility factor $\left(k_{L}\right)$ by (3) if the linear dopant drift model has been assumed. Note also that $f\left(x_{L}(t)\right)$ stands for the window function which has been used for modelling the boundary effect of the device.

$$
\frac{\mathrm{d} x_{L}(t)}{\mathrm{d} t}=k_{L} i(t) f\left(x_{L}(t)\right)
$$

On the contrary, the memcapacitance links the instantaneous flux $(\varphi(t))$ to the time integral of charge $(\sigma(t))$ by using the following relationship [5]:

$$
D_{M}(t)=\frac{\mathrm{d} \varphi(t)}{\mathrm{d} \sigma(t)}
$$

where $D_{M}(t)$ denotes the inverse memcapacitance or memelestance. In this research, the principle of memcapacitor physical operation proposed in [40] which is charge controlled has been assumed. As a result, $D_{M}(t)$ can be in terms of the minimum and maximum of $D_{M}(t)$, i.e., $D_{\text {min }}$ and $D_{\text {max }}$, and the memcapacitor's state variable $\left(x_{C}(t)\right)$ as

$$
D_{M}(t)=D_{\min }+x_{C}(t)\left(D_{\max }-D_{\min }\right)
$$

where $x_{C}(t)$ can be given in terms of $q(t)$ and the memcapacitor's mobility factor $\left(k_{C}\right)$ based on the linear dopant drift model by (6). Similarly to $f\left(x_{L}(t)\right), f\left(x_{C}(t)\right)$ denotes the window function of the memcapacitor.

$$
\frac{\mathrm{d} x_{C}(t)}{\mathrm{d} t}=k_{C} q(t) f\left(x_{C}(t)\right) \text {. }
$$

\section{The Generalization and Mathematical Modelling of Memreactance with Fractional- Order Kinetics}

After a careful consideration of (2), (3), (5), and (6), it has been found that the memreactance can be mathematically defined by using the following equation:

$$
y(t)=y_{\min }+x(t)\left(y_{\max }-y_{\min }\right),
$$

where

$$
\frac{\mathrm{d} x(t)}{\mathrm{d} t}=k J^{a}[i(t)] f(x(t)),
$$

where, $J^{a}$ [ ] stands for the integral operator of arbitrary order $a$ and $f(x(t))$ stands for the window function. It should be mentioned here that $a=0, k=k_{L}, x(t)=x_{L}(t), y(t)=\sqrt{L_{M}(t)}$, $y_{\min }=\sqrt{L_{\min }}$, and $y_{\max }=\sqrt{L_{\max }}$ if the memreactance under consideration is a meminductor. For the memcapacitor on the contrary, $a=1, \quad k=k_{C}, \quad x(t)=x_{C}(t), \quad y(t)=D_{M}(t)$, $y_{\min }=D_{\min }$, and $y_{\max }=D_{\max }$.

For the memreactance with fractional-order kinetics, we replace $\mathrm{d} / \mathrm{d} t$ in (8) by the fractional-order derivative of arbitrary real order $\alpha$, i.e., $D^{\alpha}[$ ]. Therefore, we obtain

$$
D^{\alpha}[x(t)]=k f(x(t)) z(t),
$$

where $z(t)=J^{a}[i(t)]$.

If the linear dopant drift has been assumed, the rectangular window function [2], which is linear, will be adopted. Such window function can be given by

$$
f(x(t))= \begin{cases}1, & 0 \leq x(t) \leq 1, \\ 0, & (x(t)<0) \wedge(x(t)>1) .\end{cases}
$$

Therefore, (9) becomes

$$
D^{\alpha}[x(t)]= \begin{cases}k z(t), & 0 \leq x(t) \leq 1, \\ 0, & (x(t)<0) \wedge(x(t)>1) .\end{cases}
$$

By taking $D^{a}[$ ] for both sides of this equation and keeping the above definition of $z(t)$ in mind, we have

$$
D^{\alpha+a}[x(t)]= \begin{cases}k i(t), & 0 \leq x(t) \leq 1, \\ 0, & (x(t)<0) \wedge(x(t)>1) .\end{cases}
$$

Since $a$ can be either 0 or 1 , we finally obtain

$$
D^{\beta}[x(t)]= \begin{cases}k i(t), & 0 \leq x(t) \leq 1, \\ 0, & (x(t)<0) \wedge(x(t)>1),\end{cases}
$$

where $\beta=\alpha$ for the meminductor. On the contrary, $\beta=\alpha+1$ if the memcapacitor had been considered. At this point, it can be seen that the memreactance with fractional-order 
kinetics can also be defined by using (7) but with (13) as the state equation instead of (8). By using the Riemann-Liouville fractional-order integral [8], we have

$$
x(t)= \begin{cases}0, & x(t)<0, \\ x(0)+\frac{k}{\Gamma(\beta)} \int_{0}^{t}(t-\tau)^{\beta-1} i(\tau) \mathrm{d} \tau, & 0<x(t)<1, \\ 1, & x(t) \geq 1,\end{cases}
$$

where the integral term in (14) is the Riemann-Liouville fractional-order integral. Note also that $x(0)$ and $\Gamma($ ) denote the initial value of $x(t)$ and the gamma function [41], respectively.

By using (7) and (14) and keeping in mind that the initial value of $y(t)$, i.e., $y(0)$, can be given as

$$
y(0)=y_{\min }+x(0)\left(y_{\max }-y_{\min }\right)
$$

we obtain

$$
y(t)= \begin{cases}y_{\min }, & y(t) \leq y_{\min }, \\ y(0)+\frac{k\left(y_{\max }-y_{\min }\right)}{\Gamma(\beta)} \int_{0}^{t}(t-\tau)^{\beta-1} i(\tau) \mathrm{d} \tau, & y_{\min }<y(t)<y_{\max }, \\ y_{\max }, & y(t) \geq y_{\max },\end{cases}
$$

which can be rewritten in a more compact manner in terms of the nested $\min [$ ] and $\max [$ ] similarly to the previously proposed model of the memristor with fractional-order kinetics [7] as follows:

$$
\begin{aligned}
y(t)= & \min \left[\operatorname { m a x } \left[y(0)+\frac{k\left(y_{\max }-y_{\min }\right)}{\Gamma(\beta)}\right.\right. \\
& \left.\left.\cdot \int_{0}^{t}(t-\tau)^{\beta-1} i(\tau) \mathrm{d} \tau, y_{\min }\right], y_{\max }\right] .
\end{aligned}
$$

On the contrary, if the nonlinear dopant drift model has been adopted, the analysis will become more complicated. Here, we adopt Jocklecar's window function [5] which can be given by (18) where $p \geq 1$ and $p \in\{I\}$. Note that the aforementioned rectangular window function which merely models the boundary effect in a discrete manner is approximately equivalent to Jocklecar's window function when $p$ approaches $\infty[5]$.

$$
f(x(t))=1-(2 x(t)-1)^{2 p} .
$$

By using (18), (13) becomes

$$
D^{\alpha}[x(t)]=k\left(1-(2 x(t)-1)^{2 p}\right) z(t) .
$$

As $D^{\alpha}[x(t)] \triangleq\left(\mathrm{d}^{\alpha} x(t) / \mathrm{d} t^{\alpha}\right),(19)$ can be rearranged by following [3] as

$$
\left(1-(2 x(t)-1)^{2 p}\right)^{-1} \mathrm{~d}^{\alpha} x(t)=k z(t) \mathrm{d} t^{\alpha},
$$

which is equivalent to

$$
\left(1-(2 x(t)-1)^{2 p}\right)^{-1} x^{\alpha-1}(t) \frac{\mathrm{d}^{\alpha} x(t)}{\mathrm{d}(x(t))^{\alpha}} \mathrm{d} x(t)=k z(t) t^{\alpha-1} \mathrm{~d} t .
$$

Since we define $\mathrm{d}^{\alpha} x(t) / \mathrm{d}(x(t))^{\alpha}$ as the $\alpha^{\text {th }}$ order Riemann-Liouville fractional derivative [8] of $x(t)$ with respect to $x(t)$, we have

$$
\frac{\left(1-(2 x(t)-1)^{2 p}\right)^{-1}}{\Gamma(2-\alpha)} \mathrm{d} x(t)=k z(t) t^{\alpha-1} \mathrm{~d} t
$$

which is equal to

$$
\int_{x(0)}^{x(t)}\left(1-(2 u-1)^{2 p}\right)^{-1} \mathrm{~d} u=k \Gamma(2-\alpha) \int_{0}^{t} \tau^{\alpha-1} z(\tau) \mathrm{d} \tau .
$$

If we assume $v=2 u-1$, we obtain

$$
\int_{x(0)}^{x(t)}\left(1-v^{2 p}\right)^{-1} \mathrm{~d} v=2 k \Gamma(2-\alpha) \int_{0}^{t} \tau^{\alpha-1} z(\tau) \mathrm{d} \tau .
$$

Since

$$
\int\left(1-v^{2 p}\right)^{-1} \mathrm{~d} v=\sum_{r=0}^{\infty} \frac{v^{2 p r+1}}{2 p r+1}
$$

as given in [5], the relationship between $x(t)$ and $z(t)$ can be obtained as

$$
\sum_{r=0}^{\infty} \frac{(x(t))^{2 p r+1}-(x(0))^{2 p r+1}}{2 p r+1}=2 k \Gamma(2-\alpha) \int_{0}^{t} \tau^{\alpha-1} z(\tau) \mathrm{d} \tau .
$$

As it can be, respectively, seen from (7) that $x(t)=\left(\left(y(t)-y_{\min }\right) /\left(y_{\max }-y_{\min }\right)\right), \quad$ at $x(0)=((y(0)-$ $\left.\left.y_{\min }\right) /\left(y_{\max }-y_{\min }\right)\right)$, the relationship between $y(t)$ and $z(t)$ can be finally obtained as follows: 


$$
\sum_{r=0}^{\infty} \frac{\left(\left(y(t)-y_{\min }\right) /\left(y_{\max }-y_{\min }\right)\right)^{2 p r+1}-\left(\left(y(0)-y_{\min }\right) /\left(y_{\max }-y_{\min }\right)\right)^{2 p r+1}}{2 p r+1}=2 k \Gamma(2-\alpha) \int_{0}^{t} \tau^{\alpha-1} z(\tau) \mathrm{d} \tau
$$

When $p=1$ which means that the dopant drift is highly nonlinear [5] as the degree of nonlinearity is inversely proportional to $p$ and $p \geq 1$, (26) becomes

$$
\sum_{r=0}^{\infty} \frac{x^{2 r+1}(t)}{2 r+1}-\sum_{r=0}^{\infty} \frac{x^{2 r+1}(0)}{2 r+1}=2 k \Gamma(2-\alpha) \int_{0}^{t} \tau^{\alpha-1} z(\tau) \mathrm{d} \tau
$$

which is equivalent to

$$
\tanh ^{-1}[x(t)]-\tanh ^{-1}[x(0)]=2 k \Gamma(2-\alpha) \int_{0}^{t} \tau^{\alpha-1} z(\tau) \mathrm{d} \tau
$$

Therefore, we have the following expression of $x(t)$ :

$$
x(t)=\tanh \left[\tanh ^{-1}[x(0)]+2 k \Gamma(2-\alpha) \int_{0}^{t} \tau^{\alpha-1} z(\tau) \mathrm{d} \tau\right] .
$$

By combining (7) and (30) and keeping the above expression of $x(0)$ in mind, the following expression of $y(t)$ can be obtained:

$$
\begin{aligned}
y(t)= & y_{\min }+\left(\operatorname { t a n h } \left[\tanh ^{-1}\left[\frac{y(0)-y_{\min }}{y_{\max }-y_{\min }}\right]\right.\right. \\
& \left.\left.+2 k \Gamma(2-\alpha) \int_{0}^{t} \tau^{\alpha-1} z(\tau) \mathrm{d} \tau\right]\right)\left(y_{\max }-y_{\min }\right) .
\end{aligned}
$$

However, it is very hard to obtain the exact expression of $y(t)$ like (31) when $p>1$ because it is hard to derive the exact expression of $x(t)$ like (30). As an example for illustration, we let $p=2$. Thus by using (26), we have

$$
\sum_{r=0}^{\infty} \frac{x^{4 r+1}(t)}{4 r+1}-\sum_{r=0}^{\infty} \frac{x^{4 r+1}(0)}{4 r+1}=2 k \Gamma(2-\alpha) \int_{0}^{t} \tau^{\alpha-1} z(\tau) \mathrm{d} \tau,
$$

which is equivalent to

$$
\begin{aligned}
\tan ^{-1} & (2 x(t)-1)-\tanh ^{-1}(2 x(t)-1) \\
= & 4 k \Gamma(2-\alpha) \int_{0}^{t} \tau^{\alpha-1} z(\tau) \mathrm{d} \tau+\tan ^{-1}(2 x(0)-1) \\
& \quad-\tanh ^{-1}(2 x(0)-1)
\end{aligned}
$$

Obviously, it can be seen that $x(t)$ is very hard to be analytically obtained. More complexity can be expected if larger values of $p$ have been assumed.

\section{Meminductance, Memcapacitance, and Behavioral Analysis}

By using our mathematical model, meminductance and memcapacitance of the fractional-order kinetic memreactance excited by various waveforms can be determined and the behavioral analysis can be analyzed where the DC waveform will be firstly considered followed by the AC ones as will be presented in the following sections.

4.1. DC Waveform. Mathematically, the DC waveform can be defined as $i(t)=I_{\mathrm{DC}} u(t)$, where $I_{\mathrm{DC}}$ and $u(t)$ denote the magnitude of the waveform and the unit step function. If we assume the linear dopant drift which yields a linear proportional relationship between the rate of expansion/contraction of the doped region and the applied current (charge) of the meminductor (memcapacitor), the resulting $\sqrt{L_{M}(t)}$ and $D_{M}(t)$ can be obtained by using (17) and by keeping in mind that $\beta=\alpha, k=k_{L}, y(t)=\sqrt{L_{M}(t)}, y_{\min }=\sqrt{L_{\min }}$, and $y_{\max }=\sqrt{L_{\min }}$ for the meminductor and $\beta=\alpha+1, k=k_{C}$, $y(t)=D_{M}(t), y_{\min }=D_{\min }$, and $y_{\max }=D_{\max }$ for the memcapacitor as follows:

$$
\begin{aligned}
& \sqrt{L_{M}(t)}=\min \left[\max \left[\sqrt{L_{M}(0)}+\frac{k_{L}\left(\sqrt{L_{\max }}-\sqrt{L_{\min }}\right) I_{\mathrm{DC}} t^{\alpha}}{\Gamma(\alpha+1)}, \sqrt{L_{\min }}\right], \sqrt{L_{\max }}\right] \\
& D_{M}(t)=\min \left[\max \left[D_{M}(0)+\frac{k_{C}\left(D_{\max }-D_{\min }\right) I_{\mathrm{DC}} t^{\alpha+1}}{\Gamma(\alpha+2)}, D_{\min }\right], D_{\max }\right] .
\end{aligned}
$$

After obtaining $\sqrt{L_{M}(t)}$ and $D_{M}(t), L_{M}(t)$ and memcapacitance $\left(C_{M}(t)\right)$ can be, respectively, obtained as the square of $\sqrt{L_{M}(t)}$ and reciprocal of $D_{M}(t)$. By using $L_{\min }=1 \mathrm{mH}, L_{\max }=20 \mathrm{mH}, L(0)=5 \mathrm{mH}, D_{\min }=0.1 \mathrm{MF}^{-1}$, $D_{\text {max }}=0.1 \mathrm{GF}^{-1}$, and $D(0)=0.01 \mathrm{GF}^{-1}, L_{M}(t)^{\prime} \mathrm{s}$ and $C_{M}(t)$ 's with various $\alpha$ 's excited by the DC waveform can be numerically simulated, as depicted in Figures 1-4 where $I_{\mathrm{DC}}=1 \mathrm{~A}$ has been assumed in Figures 1 and 3 and $I_{\mathrm{DC}}=-1$ A has been assumed in Figures 2 and 4. Moreover, $L_{M}(t)$ 's and $C_{M}(t)$ 's simulated based on the SPICE models of the meminductor and memcapacitor $[39,40]$ with the rectangular window function have also been included in these 


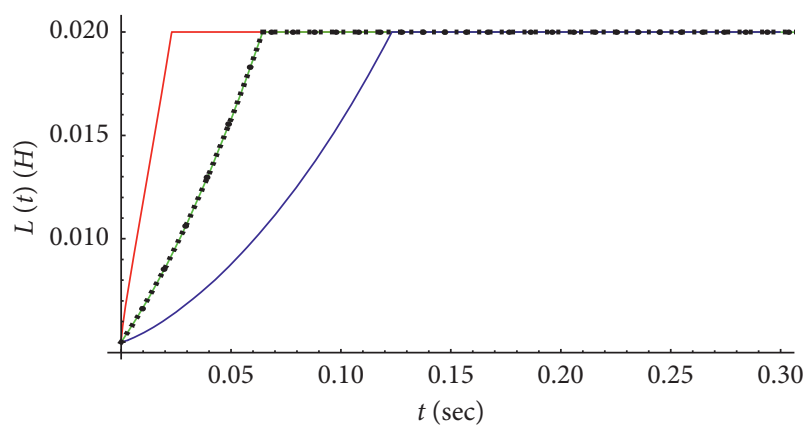

FIgURE 1: $L_{M}(t)$ due to the DC waveform with $I_{\mathrm{DC}}>0: \alpha=0.75$ (red), $\alpha=1$ (green), $\alpha=1.25$ (blue), and SPICE model (black dots).

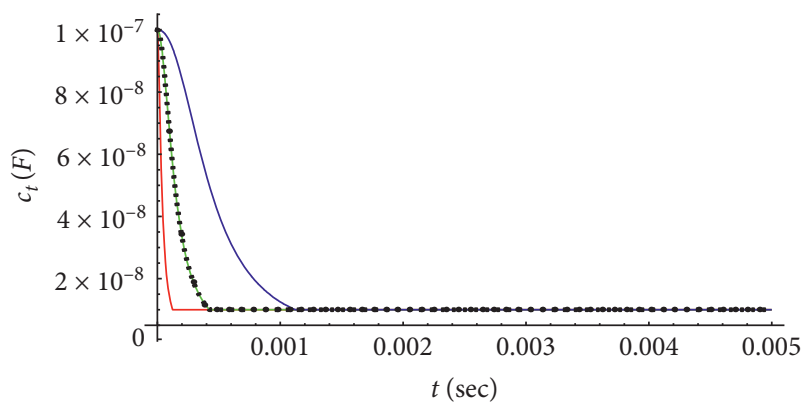

Figure 2: $C_{M}(t)$ due to the DC waveform with $I_{\mathrm{DC}}>0: \alpha=0.75$ (red), $\alpha=1$ (green), $\alpha=1.25$ (blue), and SPICE model (black dots).

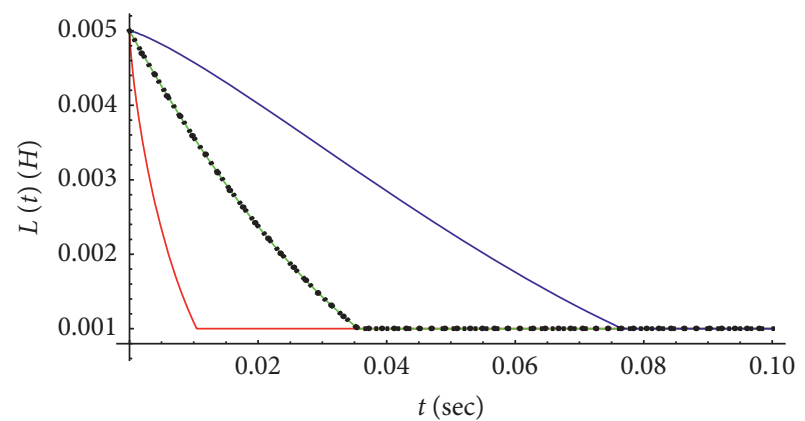

FIgURe 3: $L_{M}(t)$ due to the DC waveform with $I_{\mathrm{DC}}<0: \alpha=0.75$ (red), $\alpha=1$ (green), $\alpha=1.25$ (blue), and SPICE model (black dots).

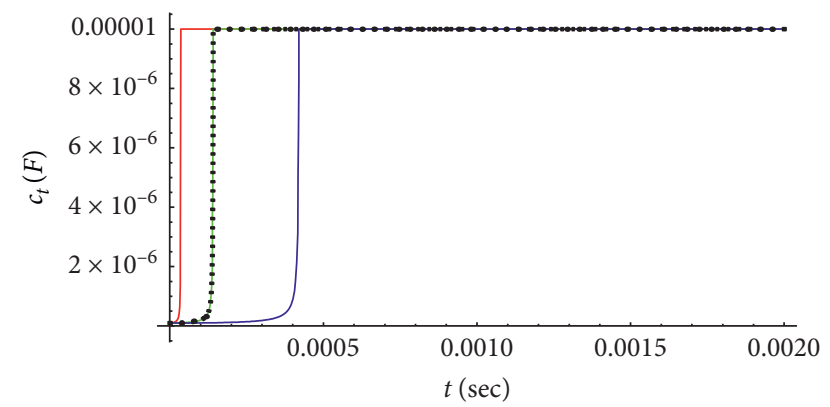

Figure 4: $C_{M}(t)$ due to the DC waveform with $I_{\mathrm{DC}}<0: \alpha=0.75$ (red), $\alpha=1$ (green), $\alpha=1.25$ (blue), and SPICE model (black dots). figures where the strong agreements between our $L_{M}(t)$ 's and $C_{M}(t)$ 's with $\alpha=1$ and their SPICE model-based benchmarks which imply the accuracy of our simulation results can be observed. These figures also show that $L_{M}(t)$ is an increasing and decreasing function of $t$ when $I_{\mathrm{DC}}>0$ and $I_{\mathrm{DC}}<0$, respectively, and vice versa for $C_{M}(t)$. However, both $L_{M}(t)$ and $C_{M}(t)$ are saturated at either $L_{\max }$ or $L_{\min }$ and $C_{\max }=1 / D_{\min }$ or $C_{\min }=1 / D_{\max }$ which are the maximum and minimum values of $C_{M}(t)$, after certain saturation times, i.e., $t_{\text {sat }, L}$ and $t_{\text {sat, } C}$, for the meminductor and memcapacitor, respectively. It has also been shown that $t_{\mathrm{sat}, L}$ and $t_{\mathrm{sat}, C}$ are directly proportional to $\alpha$.

By using the aforesaid observation, (34) and (35), we have

$$
\begin{gathered}
\sqrt{L_{M}(0)}+\frac{k_{L}\left(\sqrt{L_{\max }}-\sqrt{L_{\mathrm{min}}}\right) I_{\mathrm{DC}} t_{\mathrm{sat}, L}^{\alpha}}{\Gamma(\alpha+1)}= \begin{cases}\sqrt{L_{\max },} & I_{\mathrm{DC}}>0, \\
\sqrt{L_{\min }}, & I_{\mathrm{DC}}<0,\end{cases} \\
D_{M}(0)+\frac{k_{C}\left(D_{\max }-D_{\min }\right) I_{\mathrm{DC}} t_{\mathrm{sat}, C}^{\alpha+1}}{\Gamma(\alpha+2)}= \begin{cases}D_{\max }, & I_{\mathrm{DC}}>0, \\
D_{\min }, & I_{\mathrm{DC}}<0 .\end{cases}
\end{gathered}
$$

Therefore, $t_{\mathrm{sat}, L}$ and $t_{\mathrm{sat}, C}$ can be immediately given by

$$
\begin{aligned}
& t_{\mathrm{sat}, L}= \begin{cases}{\left[\frac{\Gamma(\alpha+1)\left(\sqrt{L_{M}(0)}-\sqrt{L_{\max }}\right)}{k_{L}\left(\sqrt{L_{\min }}-\sqrt{L_{\max }}\right) I_{\mathrm{DC}}}\right]^{1 / \alpha},} & I_{\mathrm{DC}}>0, \\
{\left[\frac{\Gamma(\alpha+1)\left(\sqrt{L_{M}(0)}-\sqrt{L_{\min }}\right)}{k_{L}\left(\sqrt{L_{\min }}-\sqrt{L_{\max }}\right) I_{\mathrm{DC}}}\right]^{1 / \alpha},} & I_{\mathrm{DC}}<0,\end{cases} \\
& t_{\text {sat }, C}= \begin{cases}{\left[\frac{\Gamma(\alpha+2)\left(D_{M}(0)-D_{\max }\right)}{k_{C}\left(D_{\min }-D_{\max }\right) I_{\mathrm{DC}}}\right]^{1 /(\alpha+1),},} & I_{\mathrm{DC}}>0, \\
{\left[\frac{\Gamma(\alpha+2)\left(D_{M}(0)-D_{\min }\right)}{k_{C}\left(D_{\min }-D_{\max }\right) I_{\mathrm{DC}}}\right]^{1 /(\alpha+1)},} & I_{\mathrm{DC}}<0,\end{cases}
\end{aligned}
$$

which shows that they are proportional to the size of difference between the initial and saturated memreactance values which can be either maxima or minima. So, both $t_{\text {sat }, L}$ and $t_{\text {sat, } C}$ reach their maximum values given, respectively, by $t_{\text {sat }, L, M}$ and $t_{\text {sat, } C, M}$, if and only if $L_{M}(0)$ and $D_{M}(0)$ reach their possible peak values given by either $L_{\min }$ and $D_{\min }$ when $I_{\mathrm{DC}}>0$ or $L_{\max }$ and $D_{\max }$ when $I_{\mathrm{DC}}<0$. Thus, $t_{\mathrm{sat}, L, M}$ and $t_{\text {sat,C,M }}$ can be found as

$$
\begin{aligned}
t_{\text {sat }, L, M} & =\left[\frac{\Gamma(\alpha+1)}{k_{L}\left|I_{\mathrm{DC}}\right|}\right]^{1 / \alpha}, \\
t_{\text {sat }, C, \text { MAX }} & =\left[\frac{\Gamma(\alpha+2)}{k_{C}\left|I_{\mathrm{DC}}\right|}\right]^{1 /(\alpha+1)} .
\end{aligned}
$$

Now, let us assume the nonlinear dopant drift which in turn leads to a nonlinear relationship between such a rate of expansion/contraction of the doped region and the current (charge) of the meminductor (memcapacitor). By using (27), the following $\sqrt{L_{M}(t)}$ and $D_{M}(t)$ with arbitrary $p$ can be obtained: 


$$
\begin{gathered}
\sum_{r=0}^{\infty} \frac{\left(\left(\sqrt{L_{M}(t)}-\sqrt{L_{\min }}\right) /\left(\sqrt{L_{\max }}-\sqrt{L_{\min }}\right)\right)^{2 p r+1}-\left(\left(\sqrt{L_{M}(0)}-\sqrt{L_{\min }}\right) /\left(\sqrt{L_{\max }}-\sqrt{L_{\min }}\right)\right)^{2 p r+1}}{2 p r+1}=\frac{2 k_{L} I_{\mathrm{DC}} \Gamma(2-\alpha) t^{\alpha}}{\alpha} \\
\sum_{r=0}^{\infty} \frac{\left(\left(D_{M}(t)-D_{\min }\right) /\left(D_{\max }-D_{\min }\right)\right)^{2 p r+1}-\left(\left(D_{M}(0)-D_{\min }\right) /\left(D_{\max }-D_{\min }\right)\right)^{2 p r+1}}{2 p r+1}=\frac{2 k_{C} \Gamma(2-\alpha) I_{\mathrm{DC}} t^{\alpha+1}}{\alpha+1} .
\end{gathered}
$$

If we let $p=1$, the resulting $\sqrt{L_{M}(t)}$ and $D_{M}(t)$ can be obtained by using (31) as follows:

$$
\begin{aligned}
& \sqrt{L_{M}(t)}=\sqrt{L_{\min }}+\left(\tanh \left[\tanh ^{-1}\left[\frac{\sqrt{L_{M}(0)}-\sqrt{L_{\min }}}{\sqrt{L_{\max }}-\sqrt{L_{\min }}}\right]+\frac{2 k_{L} I_{\mathrm{DC}} \Gamma(2-\alpha) t^{\alpha}}{\alpha}\right]\right)\left(\sqrt{L_{\max }}-\sqrt{L_{\min }}\right) \\
& D_{M}(t)=D_{\text {min }}+\left(\tanh \left[\tanh ^{-1}\left[\frac{D_{M}(0)-D_{\min }}{D_{\max }-D_{\min }}\right]+\frac{2 k_{C} \Gamma(2-\alpha) I_{\mathrm{DC}} t^{\alpha+1}}{\alpha+1}\right]\right)\left(D_{\max }-D_{\min }\right)
\end{aligned}
$$

As previously done for the conventional memcapacitor with integer kinetic transport [5], we assume that the memreactance with the nonlinear dopant drift is saturated at either $x(t)=x_{\text {on }}$, i.e., $y(t)=y_{\text {sat,on }}$, or $x(t)=x_{\text {off, }}$ i.e., $y(t)=$ $y_{\text {sat,off. }}$ By using (44) and keeping in mind that $x_{\mathrm{on}}=x_{\mathrm{on}, L}$, $x_{\text {off }}=x_{\text {off }, L}, \quad y_{\text {sat,on }}=\sqrt{L_{\text {sat,on }}}$, and $y_{\text {sat,off }}=\sqrt{L_{\text {sat,off }}}$ for the meminductor, the following nonlinear dopant drift modelbased saturation time of the meminductor can be obtained:

$$
T_{\mathrm{sat}, L}= \begin{cases}{\left[\frac{\alpha}{2 k_{L} I_{\mathrm{DC}} \Gamma(2-\alpha)}\left[\tanh ^{-1}\left[\frac{\sqrt{L_{\mathrm{sat}, \text { off }}}-\sqrt{L_{\min }}}{\sqrt{L_{\max }}-\sqrt{L_{\min }}}\right]-\tanh ^{-1}\left[\frac{\sqrt{L_{M}(0)}-\sqrt{L_{\min }}}{\sqrt{L_{\max }}-\sqrt{L_{\min }}}\right]\right]\right]^{1 / \alpha},} & I_{\mathrm{DC}}>0, \\ {\left[\frac{\alpha}{2 k_{L} I_{\mathrm{DC}} \Gamma(2-\alpha)} \tanh ^{-1}\left[\frac{\sqrt{L_{\mathrm{sat}, \text { on }}}-\sqrt{L_{\min }}}{\sqrt{L_{\max }}-\sqrt{L_{\min }}}\right]-\tanh ^{-1}\left[\frac{\sqrt{L_{M}(0)}-\sqrt{L_{\min }}}{\sqrt{L_{\max }}-\sqrt{L_{\min }}}\right]\right]^{1 / \alpha},} & I_{\mathrm{DC}}<0 .\end{cases}
$$

On the contrary, the nonlinear dopant drift model-based saturation time of the memcapacitor can be derived by using
(45) and keeping in mind that $x_{\mathrm{on}}=x_{\mathrm{on}, C}, x_{\mathrm{off}}=x_{\mathrm{off}, C}$, $y_{\text {sat,on }}=D_{\text {sat,on }}$, and $y_{\text {sat,off }}=D_{\text {sat,off }}$ as

$$
T_{\text {sat }, C}=\left\{\begin{array}{l}
{\left[\frac{\alpha+1}{2 k_{C} \Gamma(2-\alpha) I_{\mathrm{DC}}}\left[\tanh ^{-1}\left[\frac{D_{\mathrm{sat}, \text { off }}-D_{\min }}{D_{\max }-D_{\min }}\right]-\tanh ^{-1}\left[\frac{D_{M}(0)-D_{\min }}{D_{\max }-D_{\min }}\right]\right]\right]^{1 /(\alpha+1)}, \quad I_{\mathrm{DC}}>0,} \\
{\left[\frac{\alpha+1}{2 k_{C} \Gamma(2-\alpha) I_{\mathrm{DC}}}\left[\tanh ^{-1}\left[\frac{D_{\mathrm{sat}, \text { on }}-D_{\min }}{D_{\max }-D_{\min }}\right]-\tanh ^{-1}\left[\frac{D_{M}(0)-D_{\min }}{D_{\max }-D_{\min }}\right]\right]\right]^{1 /(\alpha+1)}, \quad I_{\mathrm{DC}}<0 .}
\end{array}\right.
$$

Since the maximum saturation time occurred when the memreactance is saturated at either $y(t)=y_{\text {on }}$ given $y(0)=$ $y_{\text {off }}$ or $y(t)=y_{\text {off }}$ given $y(0)=y_{\text {on }}$, the maximal values of $T_{\text {sat }, L}$ and $T_{\text {sat, } C}$ can be, respectively, given by 


$$
\begin{aligned}
& T_{\text {sat }, L, M}= \begin{cases}{\left[\frac{\alpha}{2 k_{L} I_{\mathrm{DC}} \Gamma(2-\alpha)}\left[\tanh ^{-1}\left[\frac{\sqrt{L_{\mathrm{sat}, \text { off }}}-\sqrt{L_{\min }}}{\sqrt{L_{\mathrm{max}}}-\sqrt{L_{\min }}}\right]-\tanh ^{-1}\left[\frac{\sqrt{L_{\mathrm{sat}, \mathrm{on}}}-\sqrt{L_{\min }}}{\sqrt{L_{\max }}-\sqrt{L_{\min }}}\right]\right]\right]^{1 / \alpha},} & I_{\mathrm{DC}}>0, \\
{\left[\frac{\alpha}{2 k_{L} I_{\mathrm{DC}} \Gamma(2-\alpha)} \tanh ^{-1}\left[\frac{\sqrt{L_{\mathrm{sat}, \text { on }}}-\sqrt{L_{\min }}}{\sqrt{L_{\max }}-\sqrt{L_{\min }}}\right]-\tanh ^{-1}\left[\frac{\sqrt{L_{\mathrm{sat}, \text { off }}}-\sqrt{L_{\min }}}{\sqrt{L_{\max }}-\sqrt{L_{\min }}}\right]\right]^{1 / \alpha},} & I_{\mathrm{DC}}<0,\end{cases} \\
& T_{\text {sat }, C, M}= \begin{cases}{\left[\frac{\alpha+1}{2 k_{C} \Gamma(2-\alpha) I_{\mathrm{DC}}}\left[\tanh ^{-1}\left[\frac{D_{\text {sat,off }}-D_{\min }}{D_{\max }-D_{\min }}\right]-\tanh ^{-1}\left[\frac{D_{\text {sat,on }}-D_{\min }}{D_{\max }-D_{\min }}\right]\right]\right]^{1 /(\alpha+1)},} & I_{\mathrm{DC}}>0, \\
{\left[\frac{\alpha+1}{2 k_{C} \Gamma(2-\alpha) I_{\mathrm{DC}}}\left[\tanh ^{-1}\left[\frac{D_{\text {sat,on }}-D_{\min }}{D_{\max }-D_{\min }}\right]-\tanh ^{-1}\left[\frac{D_{\text {sat,off }}-D_{\min }}{D_{\max }-D_{\min }}\right]\right]\right]^{1 /(\alpha+1)},} & I_{\mathrm{DC}}<0,\end{cases}
\end{aligned}
$$

which can be simplified as

$$
\begin{aligned}
T_{\text {sat }, L, M}= & {\left[\frac { \alpha } { 2 k _ { L } | I _ { \mathrm { DC } } | \Gamma ( 2 - \alpha ) } \left[\tanh ^{-1}\left[x_{L, \text { off }}\right]\right.\right.} \\
& \left.\left.-\tanh ^{-1}\left[x_{L, \text { on }}\right]\right]\right]^{1 / \alpha}, \\
T_{\text {sat }, C, M=}= & {\left[\frac { \alpha + 1 } { 2 k _ { C } \Gamma ( 2 - \alpha ) | I _ { \mathrm { DC } } | } \left[\tanh ^{-1}\left[x_{C, \mathrm{off}}\right]\right.\right.} \\
& \left.\left.-\tanh ^{-1}\left[x_{C, \mathrm{on}}\right]\right]\right]^{1 /(\alpha+1)} .
\end{aligned}
$$

At this point, we simulate $t_{\mathrm{sat}, L, M}, t_{\mathrm{sat}, C, M}, T_{\mathrm{sat}, L, M}$, and $T_{\text {sat, } C, M}$ with respect to $I_{\mathrm{DC}}$ by assuming that $x_{\mathrm{on}, C}=x_{\mathrm{on}, L}=0.01$ and $x_{\mathrm{off}, C}=x_{\mathrm{off}, L}=0.99$ where other parameters similar to those of the simulation of $L_{M}(t)$ and $C_{M}(t)$ have been adopted. The results are depicted in Figures 5 and 6 which show that these saturation times are directly proportional to $\alpha$ but inversely proportional to the magnitude of $I_{\mathrm{DC}}$. It can also be seen that the memreactance with the nonlinear dopant drift except the memcapacitor with $\alpha>1$ takes longer time to reach saturation.

Before we proceed to the subsequent section, it should be mentioned here that

$$
\begin{aligned}
& \frac{T_{\text {sat }, M, L}}{t_{\text {sat }, M, L}}=\left[\frac{\tanh ^{-1}\left[x_{L, \text { off }}\right]-\tanh ^{-1}\left[x_{L, \text { on }}\right]}{2 \Gamma(\alpha) \Gamma(2-\alpha)}\right]^{1 / \alpha}, \\
& \frac{T_{\text {sat }, M, C}}{t_{\text {sat }, M, C}}=\left[\frac{\tanh ^{-1}\left[x_{C, \text { off }}\right]-\tanh ^{-1}\left[x_{C, \text { on }}\right]}{2 \Gamma(2-\alpha) \Gamma(\alpha+1)}\right]^{1 /(\alpha+1)} .
\end{aligned}
$$

4.2. Periodic Waveforms. For the sinusoidal waveform with arbitrary phase $(\theta)$, i.e., $i(t)=I_{m} \sin (\omega t+\theta)$, where $I_{m}$ and $\omega$, respectively, denote its peak value and angular frequency, $\sqrt{L_{M}(t)}$ with the linear dopant drift can be given by using (17) with $\beta=\alpha, k=k_{L}, y(t)=\sqrt{L_{M}(t)}, y_{\min }=\sqrt{L_{\text {min }}}$, and $y_{\max }=\sqrt{L_{\min }}$ as

$$
\begin{aligned}
\sqrt{L_{M}(t)}= & \min \left[\operatorname { m a x } \left[\sqrt{L_{M}(0)}-\frac{k_{L}\left(\sqrt{L_{\min }}-\sqrt{L_{\max }}\right) I_{m} t^{\alpha}}{\Gamma(\alpha+1)}\right.\right. \\
& \cdot\left[\sin (\theta)_{1} F_{2}\left(1 ; \frac{\alpha}{2}+\frac{1}{2}, \frac{\alpha}{2}+1 ;-\frac{1}{4}(\omega t)^{2}\right)\right. \\
& \left.+\frac{\omega t \cos (\theta)}{\alpha+1} F_{2}\left(1 ; \frac{\alpha}{2}+1, \frac{\alpha}{2}+\frac{3}{2} ;-\frac{1}{4}(\omega t)^{2}\right)\right], \\
& \left.\left.\sqrt{L_{\min }}\right], \sqrt{L_{\max }}\right]
\end{aligned}
$$

where ${ }_{1} F_{2}(; ; ;)$ denotes a generalized hypergeometric function with $p=1$ and $q=2$ [42].

At the steady state, $\sqrt{L_{M}(t)}$ is reduced to the following equation which can be given by using our model and the asymptotic approximation of the sinusoidal function's fractional-order integration [43]:

$$
\begin{aligned}
\sqrt{L_{M}(t)}= & \min \left[\operatorname { m a x } \left[\sqrt{L_{M}(0)}-\frac{k_{L}\left(\sqrt{L_{\min }}-\sqrt{L_{\max }}\right) I_{m}}{\omega^{\alpha}}\right.\right. \\
& \cdot\left[\cos \left(\theta+\frac{1-\alpha}{2} \pi\right)-\cos \left(\omega t+\theta+\frac{1-\alpha}{2} \pi\right)\right], \\
& \left.\left.\sqrt{L_{\min }}\right], \sqrt{L_{\max }}\right] .
\end{aligned}
$$

By using (53) with $\theta=\pi / 2 \mathrm{rad}, L_{\min }=1 \mathrm{mH}$, $L_{\max }=20 \mathrm{mH}$, and $L(0)=5 \mathrm{mH}$, we can simulate the $\rho(t)-q(t)$ and $\varphi(t)-i(t)$ characteristics of the fractional-order kinetic meminductor with various $\alpha$ 's, as depicted in Figures 7-10 where $I_{m}=10 \mathrm{~mA}$ has been assumed in Figures 7 and 9. On the contrary, $I_{m}=-10 \mathrm{~mA}$ has been adopted in Figures 8 and 10. Moreover, the vertical axes of these figures have been scaled up by 100 for visibility. From Figures 7 and 8 , it can be seen that the one-to-one correspondence between $\rho(t)$ and $q(t)$ ceased to be existed when $\alpha \neq 1$ which means that the constitutive relation of the meminductor is ambiguous when its kinetic transport is of fractional order. Therefore, the unambiguous constitutive relation which is one of the basic fingerprints of the meminductor [39] cannot be preserved by the fractional-order domain generalization. 


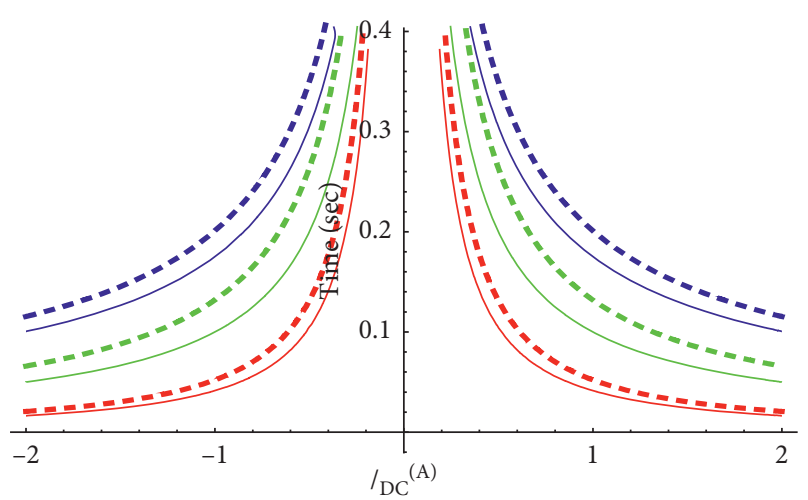

Figure 5: $t_{\text {sat }, L, M}$ (line) and $T_{\text {sat }, L, M}$ (dashed): $\alpha=0.75$ (red), $\alpha=1$ (green), and $\alpha=1.25$ (blue).

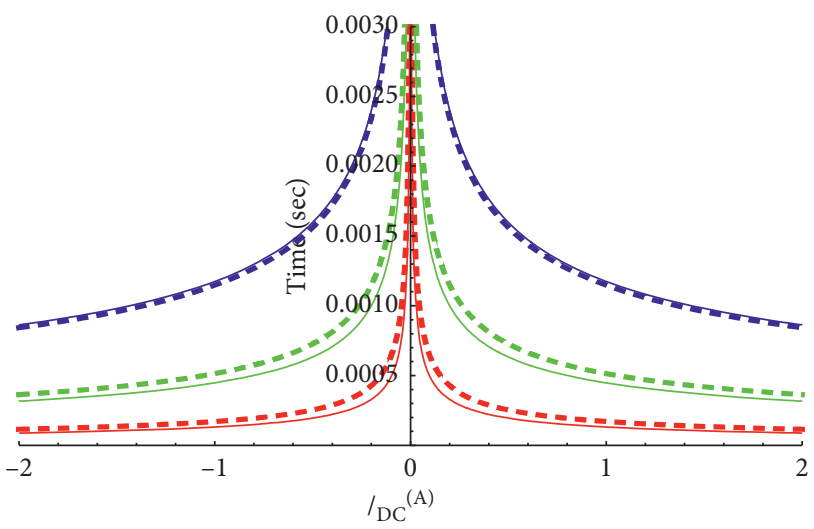

Figure 6: $t_{\text {sat }, C, M}$ (line) and $T_{\text {sat }, C, M}$ (dashed): $\alpha=0.75$ (red), $\alpha=1$ (green), and $\alpha=1.25$ (blue).

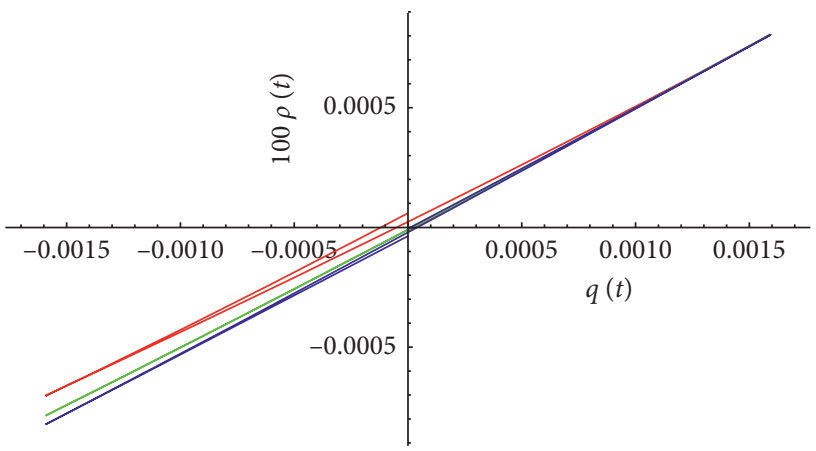

FIgURE 7: $\rho(t)-q(t)$ of the meminductor under the periodic waveform with $I_{m}>0: \alpha=0.75$ (red), $\alpha=1$ (green), and $\alpha=1.25$ (blue).

However, despite the asymmetricities, the pinched hysteresis loop in the $\varphi(t)-i(t)$ characteristic which is also a basic fingerprint of the meminductor can be preserved by the fractional-order domain generalization as can be seen from Figures 9 and 10 where the strong agreements between the $\varphi(t)-i(t)$ Lissajous curve of the device with $\alpha=1$ and the SPICE meminductor model counterpart with rectangular window function that verify our results can be observed. Moreover, it can be observed that the lobe area of the

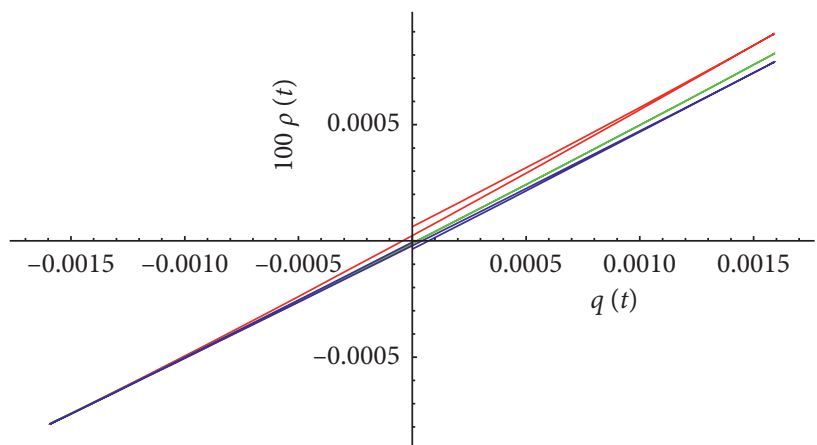

FIGURE 8: $\rho(t)-q(t)$ of the meminductor under the periodic waveform with $I_{m}<0: \alpha=0.75$ (red), $\alpha=1$ (green), and $\alpha=1.25$ (blue).

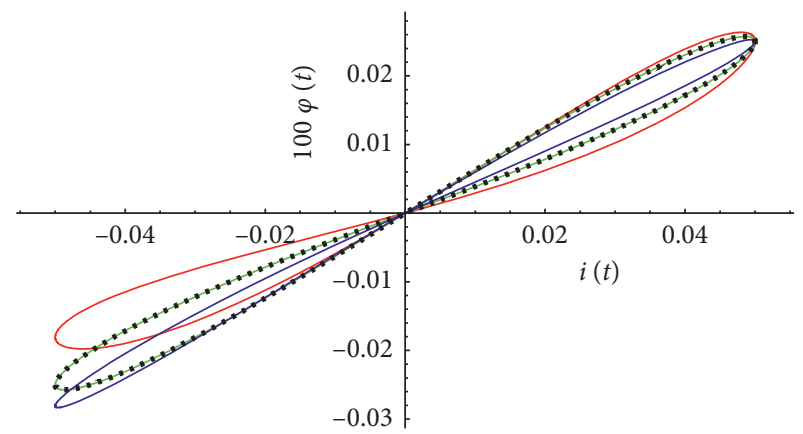

FIgURE 9: $\varphi(t)-i(t)$ of the meminductor under the periodic waveform with $I_{m}>0$ : $\alpha=0.75$ (red), $\alpha=1$ (green), $\alpha=1.25$ (blue), and SPICE model (black dots).

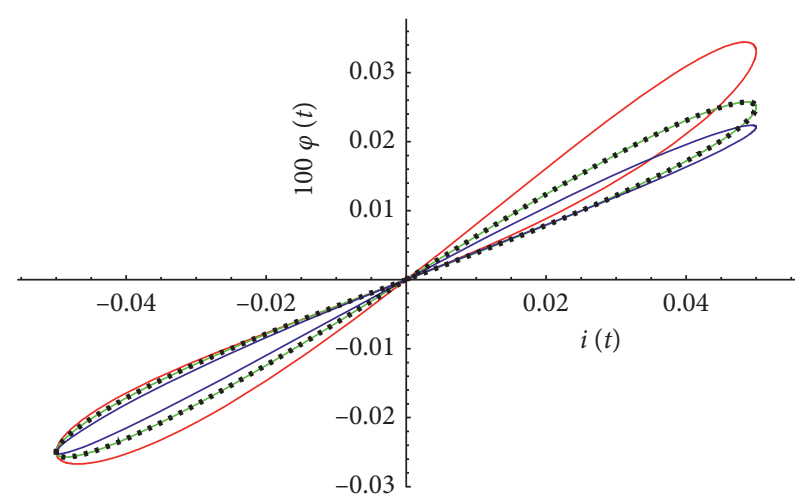

Figure 10: $\varphi(t)-i(t)$ of the meminductor under the periodic waveform with $I_{m}<0$ : $\alpha=0.75$ (red), $\alpha=1$ (green), $\alpha=1.25$ (blue), and SPICE model (black dots).

pinched hysteresis loop which refers to the memory effect and linearity is inversely proportional to $\alpha$. Therefore, more memory effect and less linearity can be obtained by using the meminductor with fractional-order kinetics of lower $\alpha$, and vice versa.

When the kinetic transport is fractional, the meminductor becomes unbalanced unless $\alpha=1$ as it has nonzero average power consumption caused by nonzero stored energy after an integer number of periods. In order to illustrate this issue, the areas of the upper lobe and lower lobe of the 
$\varphi(t)$-i $(t)$ Lissajous curve, i.e., $A_{\mathrm{UL}}$ and $A_{\mathrm{LL}}$, must be firstly calculated. For doing so, we use the following equations:

$$
\begin{aligned}
& A_{\mathrm{UL}}=\int_{-\theta / \omega}^{(\pi-\theta) / \omega} \varphi(t) \mathrm{d} i(t), \\
& A_{\mathrm{LL}}=\int_{(\pi-\theta) / \omega}^{(2 \pi-\theta) / \omega} \varphi(t) \mathrm{d} i(t) .
\end{aligned}
$$

Since $\varphi(t)=L_{M}(t) i(t)$, where $i(t)=I_{m} \sin (\omega t+\theta), A_{\mathrm{UL}}$ and $A_{\mathrm{LL}}$ can be obtained by using (53)-(55) as follows:

$$
\begin{aligned}
A_{\mathrm{UL}}= & -\frac{k I_{m}^{3} \pi}{12 \omega^{2 \alpha}} \sin \left(\frac{\alpha \pi}{2}\right)\left(\sqrt{L_{\max }}-\sqrt{L_{\min }}\right)^{2} \\
& \times\left[16 \omega^{\alpha} \sqrt{L_{M}(0)}+k I_{m}\left(\sqrt{L_{\max }}-\sqrt{L_{\min }}\right)\left(3 \pi \cos \left(\frac{\alpha \pi}{2}\right)\right.\right. \\
- & \left.\left.16 \sin \left(\frac{\alpha \pi}{2}-\theta\right)\right)\right], \\
A_{\mathrm{LL}}= & \frac{k I_{m}^{3} \pi}{12 \omega^{2 \alpha}} \sin \left(\frac{\alpha \pi}{2}\right)\left(\sqrt{L_{\max }}-\sqrt{L_{\min }}\right)^{2} \\
& \times\left[16 \omega^{\alpha} \sqrt{L_{M}(0)}+k I_{m}\left(\sqrt{L_{\max }}-\sqrt{L_{\min }}\right)\right. \\
& \left.\cdot\left(16 \sin \left(\frac{\alpha \pi}{2}-\theta\right)-3 \pi \cos \left(\frac{\alpha \pi}{2}\right)\right)\right]
\end{aligned}
$$

Therefore, $A_{\mathrm{UL}}+A_{\mathrm{LL}}$ can be immediately found as

$$
A_{\mathrm{UL}}+A_{\mathrm{LL}}=-\frac{k^{2} I_{m}^{4} \pi}{4 \omega^{2 \alpha}} \sin (\alpha \pi)\left(\sqrt{L_{\max }}-\sqrt{L_{\min }}\right)^{2} \text {. }
$$

Since $A_{\mathrm{UL}}$ and $A_{\mathrm{LL}}$ are, respectively, referred to as the energy intake and energy dissipated during the positive and negative half cycles, $A_{\mathrm{UL}}+A_{\mathrm{LL}}$ refers to the total stored energy after a period and its multiple is referred to as the aforesaid net stored energy after an integer number of periods. $A_{\mathrm{UL}}+A_{\mathrm{LL}} \neq 0$, and so do its multiple and such net stored energy; thus, nonzero average power consumption is obtained and the aforementioned unbalance occurred. However, it can be seen from (51) that $A_{\mathrm{UL}}+A_{\mathrm{LL}}=0$ and so do its multiple, the net stored energy, and the average power consumption if we let $\alpha=1$. Therefore, the balance can be achieved with this specific value of $\alpha$. It can also be seen from (56)-(58) that the total stored energy after a period, net stored energy after an integer number of periods, and average power consumption are independent of $\theta$ despite the energy intake and energy dissipated during the positive and negative half cycles are dependent.

Despite employing fractional-order kinetic transport, the meminductor still has identical time instants at which $\varphi(t)$ and $i(t)$ cross zero levels; that is, the fractional-order domain generalization preserves this basic fingerprint of the meminductor. For illustration, we simulate $\varphi(t)$ of the fractional-order meminductor with various $\alpha$ 's, as depicted in Figures 11 and 12 where $i(t)$ has also been included and $\varphi(t)$ 's have been multiplied by 100 for visibility.

From Figures 11 and 12 where $\theta=\pi / 2 \mathrm{rad}, L_{\min }=1 \mathrm{mH}$, $L_{\text {max }}=20 \mathrm{mH}$, and $L(0)=5 \mathrm{mH}$ have also been assumed, it can be seen that time instants at which both $\varphi(t)$ and $i(t)$ cross zero levels are identical. Without regarding any specific device, we commonly denote these time instants by $t_{x 0}$. Since $i(t)=I_{m} \sin (\omega t+\theta), t_{x 0}$ can be obtained by

$$
t_{x 0}=\frac{n \pi-\theta}{\omega}
$$

where $n=\{1,2,3, \ldots\}$.

Now, the effects of the nonlinear dopant drift will be studied. By using (27), the resulting $\sqrt{L_{M}(t)}$ with arbitrary $p$ can be found as

$$
\begin{aligned}
& \sum_{r=0}^{\infty} \frac{\left(\left(\sqrt{L_{M}(t)}-\sqrt{L_{\min }}\right) /\left(\sqrt{L_{\max }}-\sqrt{L_{\min }}\right)\right)^{2 p r+1}-\left(\left(\sqrt{L_{M}(0)}-\sqrt{L_{\min }}\right) /\left(\sqrt{L_{\max }}-\sqrt{L_{\min }}\right)\right)^{2 p r+1}}{2 p r+1} \\
& \quad=j k_{L} I_{m} \Gamma(2-\alpha) \omega^{-\alpha}[\exp (j \theta) \Gamma(\alpha,-j \omega t)-\exp (-j \theta) \Gamma(\alpha, j \omega t)],
\end{aligned}
$$

where $\Gamma($ ) denotes the incomplete gamma function [44].
On the contrary, $\sqrt{L_{M}(t)}$ with $p=1$ can be obtained by using (31) as follows:

$$
\begin{aligned}
\sqrt{L_{M}(t)=} & \sqrt{L_{\min }}+\left(\operatorname { t a n h } \left[\tanh ^{-1}\left[\frac{\sqrt{L_{M}(0)}-\sqrt{L_{\min }}}{\sqrt{L_{\max }}-\sqrt{L_{\min }}}\right]+j k_{L} I_{m} \Gamma(2-\alpha) \omega^{-\alpha}[\exp (j \theta) \Gamma(\alpha,-j \omega t)\right.\right. \\
& -\exp (-j \theta) \Gamma(\alpha, j \omega t)]])\left(\sqrt{L_{\max }}-\sqrt{L_{\min }}\right) .
\end{aligned}
$$




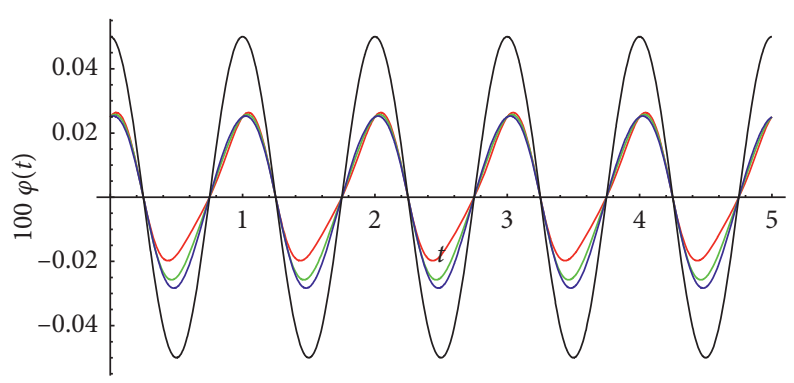

Figure 11: $\varphi(t)-i(t)$ of the meminductor under the periodic waveform with $I_{m}>0: \alpha=0.75$ (red), $\alpha=1$ (green), $\alpha=1.25$ (blue), and $i(t)$ (black).

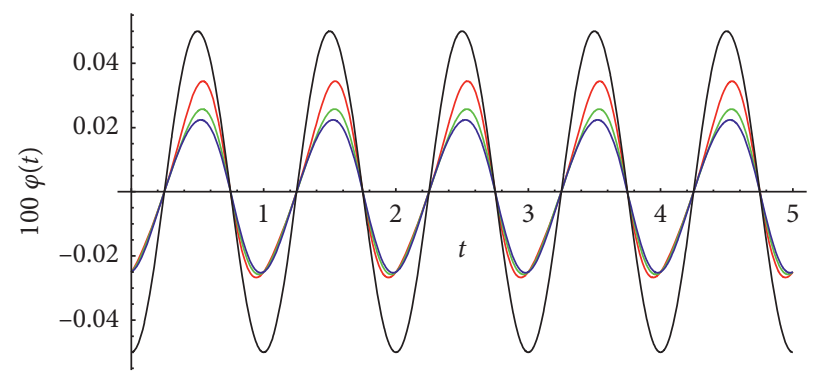

FIGURE 12: $\varphi(t)-i(t)$ of the meminductor under the periodic waveform with $I_{m}<0: \alpha=0.75$ (red), $\alpha=1$ (green), $\alpha=1.25$ (blue), and $i(t)$ (black).

If we let $\alpha$ approach $0, \int_{0}^{t} \tau^{\alpha-1} z(\tau) \mathrm{d} \tau$ approaches $\int_{0}^{t} \tau^{-1} z(\tau) \mathrm{d} \tau$. Thus by also, respectively, applying (27) and (31), we have

$$
\begin{aligned}
& \sum_{r=0}^{\infty} \frac{\left(\left(\sqrt{L_{M}(t)}-\sqrt{L_{\min }}\right) /\left(\sqrt{L_{\max }}-\sqrt{L_{\min }}\right)\right)^{2 p r+1}-\left(\left(\sqrt{L_{M}(0)}-\sqrt{L_{\min }}\right) /\left(\sqrt{L_{\max }}-\sqrt{L_{\min }}\right)\right)^{2 p r+1}}{2 p r+1} \\
& \quad=2 k_{L} I_{m} \Gamma(2-\alpha)[\sin (\theta) \operatorname{Ci}(\omega t)+\cos (\theta) \operatorname{Si}(\omega t)] \\
& \quad \sqrt{L_{M}(t)}=\sqrt{L_{\min }}+\left(\tanh \left[\tanh ^{-1}\left[\frac{\sqrt{L_{M}(0)}-\sqrt{L_{\min }}}{\sqrt{L_{\max }}-\sqrt{L_{\min }}}\right]+2 k_{L} I_{m} \Gamma(2-\alpha)[\sin (\theta) \operatorname{Ci}(\omega t)+\cos (\theta) \operatorname{Si}(\omega t)]\right]\right)\left(\sqrt{L_{\max }}-\sqrt{L_{\min }}\right)
\end{aligned}
$$

where $\mathrm{Si}(\mathrm{)}$ and $\mathrm{Ci}(\mathrm{)}$ stand for sine and cosine integral functions [45].
If $\alpha$ approaches 1 on the contrary, $\int_{0}^{t} \tau^{\alpha-1} z(\tau) \mathrm{d} \tau$ approaches $\int_{0}^{t} z(\tau) \mathrm{d} \tau$. As a result, we have

$$
\begin{aligned}
& \sum_{r=0}^{\infty} \frac{\left(\left(\sqrt{L_{M}(t)}-\sqrt{L_{\min }}\right) /\left(\sqrt{L_{\max }}-\sqrt{L_{\min }}\right)\right)^{2 p r+1}-\left(\left(\sqrt{L_{M}(0)}-\sqrt{L_{\min }}\right) /\left(\sqrt{L_{\max }}-\sqrt{L_{\min }}\right)\right)^{2 p r+1}}{2 p r+1}=-\frac{2 k_{L} I_{m} \Gamma(2-\alpha) \cos (\omega \tau+\theta)}{\omega} \\
& \sqrt{L_{M}(t)}=\sqrt{L_{\min }}+\left(\tanh \left[\tanh ^{-1}\left[\frac{\sqrt{L_{M}(0)}-\sqrt{L_{\min }}}{\sqrt{L_{\max }}-\sqrt{L_{\min }}}\right]-\frac{2 k_{L} I_{m} \Gamma(2-\alpha) \cos (\omega \tau+\theta)}{\omega}\right]\right)\left(\sqrt{L_{\max }}-\sqrt{L_{\min }}\right)
\end{aligned}
$$

With these results, we can simulate $L_{M}(t)$ with the effect of the nonlinear dopant drift as dashed curves in Figures 13 and 14 where those based on the linear dopant drift have also been included as normal curves for comparison. We also simulate the $\varphi(t)-i(t)$ characteristic, as shown in Figures 15 and 16 where the dashed curves have been adopted for being 


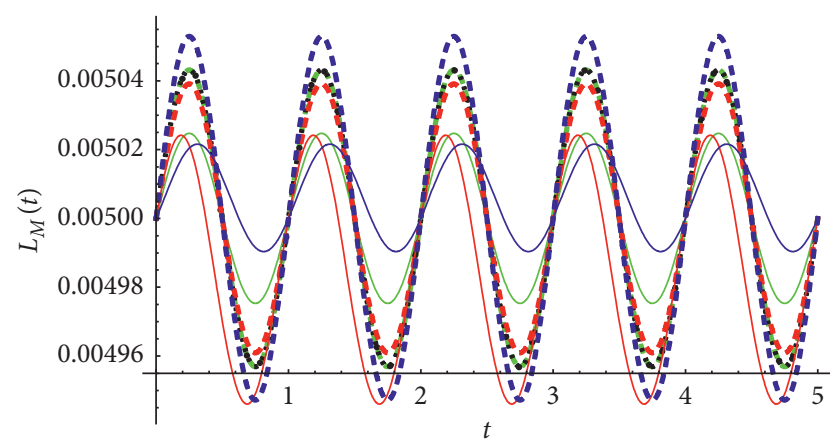

FIGURE 13: $L_{M}(t)$ of the meminductor with linear (normal curve) and nonlinear (dashed curve) dopant drifts under the periodic waveform with $I_{m}>0: \alpha=0.75$ (red), $\alpha=1$ (green), $\alpha=1.25$ (blue), and SPICE model (black).

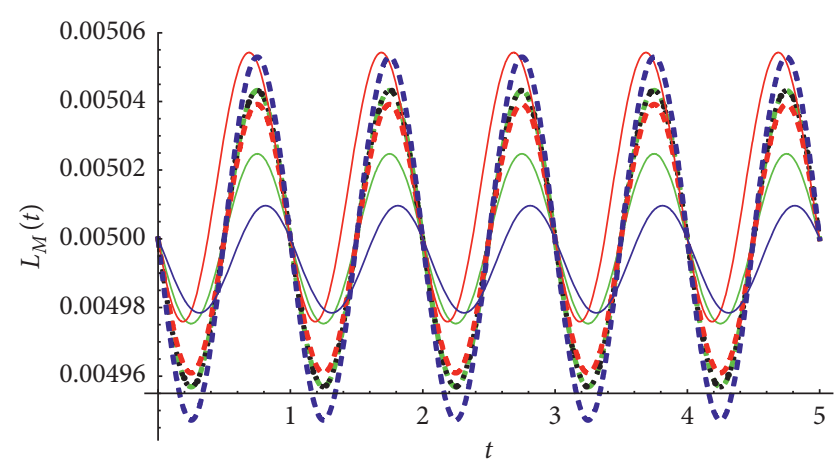

Figure 14: $L_{M}(t)$ of the meminductor with linear (normal curve) and nonlinear (dashed curve) dopant drifts under the periodic waveform with $I_{m}<0$ : $\alpha=0.75$ (red), $\alpha=1$ (green), $\alpha=1.25$ (blue), and SPICE model (black).

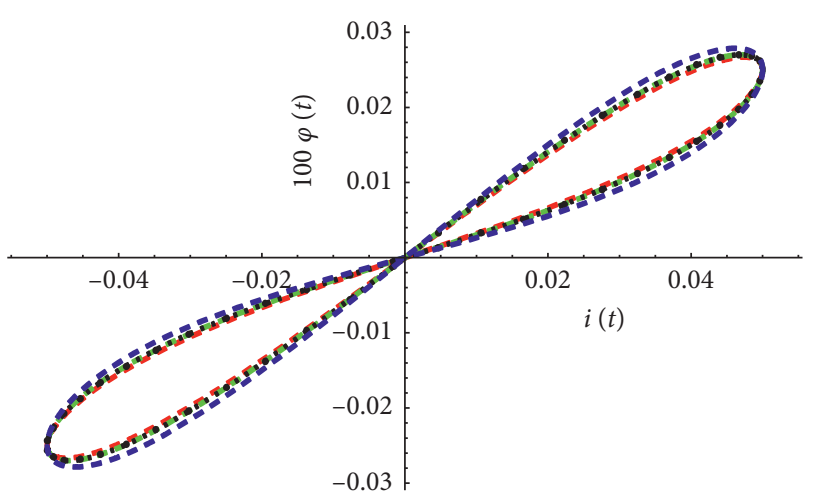

FIGURE 15: $\varphi(t)-i(t)$ of the meminductor with the nonlinear dopant drift under the periodic waveform with $I_{m}>0: \alpha=0.75$ (red), $\alpha=1$ (green), $\alpha=1.25$ (blue), and SPICE model (black dots).

distinguished from the linear dopant drift-based $\varphi(t)-i(t)$ depicted in Figures 9 and 10. Here, we choose $p=1$ where $\theta=\pi / 2 \mathrm{rad}, L_{\min }=1 \mathrm{mH}, L_{\max }=20 \mathrm{mH}$, and $L(0)=5 \mathrm{mH}$ have also been assumed. From these figures where the strong agreements between our results with the nonlinear dopant drift and those based on the SPICE model with Jocklecar's window function and $p=1$ can be observed, and Figures 9

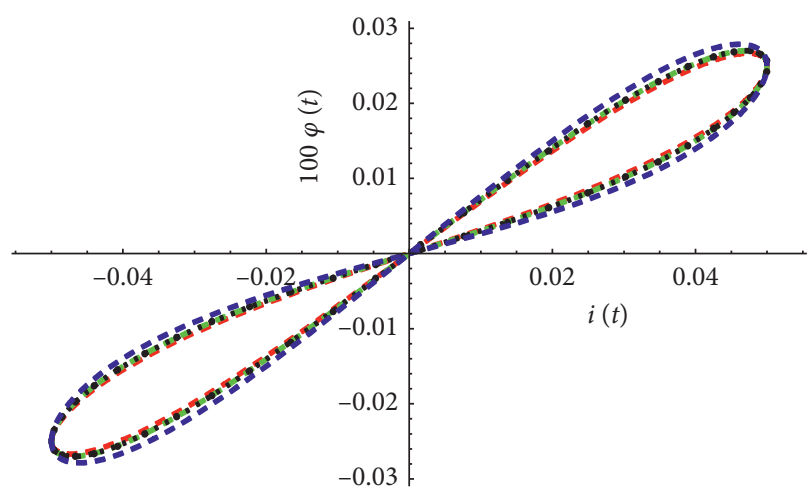

Figure 16: $\varphi(t)-i(t)$ of the meminductor with the nonlinear dopant drift under the periodic waveform with $I_{m}<0: \alpha=0.75$ (red), $\alpha=1$ (green), $\alpha=1.25$ (blue), and SPICE model (black dots).

and 10, it can be seen that the nonlinear dopant drift reduces the effects of $\alpha$ on the variation in $L_{M}(t)$ and variation in nonlinearity of the device; that is, the device is more robust to the effects of fractional kinetic transport. We have also found that the meminductor becomes more nonlinear with increasing $\alpha$ and with a more symmetric pinched hysteresis loop if the nonlinear dopant drift has been assumed for ceteris paribus.

At this point, the memcapacitor with fractional-order kinetics will be considered where cosinusoidal with arbitrary $\theta$, i.e., $i(t)=I_{m} \cos (\omega t+\theta)$, will be assumed. Assuming the linear dopant drift, $D_{M}(t)$ can be given by using (17) with $\beta=\alpha+1, k=k_{C}, y(t)=D_{M}(t), y_{\min }=D_{\min }$, and $y_{\max }=D_{\max }$ as

$$
\begin{aligned}
D_{M}(t)= & D_{M}(0)-\frac{k_{C}\left(D_{\min }-D_{\max }\right) I_{m} t^{\alpha+1}}{\Gamma(\alpha+2)} \\
& \cdot\left[\operatorname { s i n } ( \theta ) _ { 1 } F _ { 2 } \left(1 ; \frac{\alpha+1}{2}+\frac{1}{2}, \frac{\alpha+1}{2}+1 ;-\frac{1}{4}\right.\right. \\
& \left.\cdot\left(\frac{\pi}{2}-\omega t-2 \theta\right)^{2}\right)+\frac{((\pi / 2)-\omega t-2 \theta) \cos (\theta)}{\alpha+2}{ }_{1} F_{2} \\
& \left.\cdot\left(1 ; \frac{\alpha+1}{2}+1, \frac{\alpha+1}{2}+\frac{3}{2} ;-\frac{1}{4}\left(\frac{\pi}{2}-\omega t-2 \theta\right)^{2}\right)\right] .
\end{aligned}
$$

Similarly to $\sqrt{L_{M}(t)}, D_{M}(t)$ is reduced to a simplified version at the steady state given by

$$
\begin{aligned}
D_{M}(t)= & D_{M}(0)-\frac{k_{C}\left(D_{\min }-D_{\max }\right) I_{m}}{\omega^{\alpha+1}}\left[\cos \left(\theta-\frac{\alpha}{2} \pi\right)\right. \\
& \left.-\cos \left(\frac{1-\alpha}{2} \pi-(\omega t+\theta)\right)\right] .
\end{aligned}
$$

By using (65) with $\theta=0 \mathrm{rad}, \quad D_{\min }=0.1 \mathrm{MF}^{-1}$, $D_{\text {max }}=0.1 \mathrm{GF}^{-1}$, and $D(0)=0.01 \mathrm{GF}^{-1}$, we can simulate the $\varphi(t)-\sigma(t)$ and $v(t)-q(t)$ characteristics with various $\alpha$ 's, as shown in Figures $17-20$ where $I_{m}=0.1 \mu \mathrm{A}$ has been assumed in Figures 17 and 19. On the contrary, $I_{m}=-0.1 \mu \mathrm{A}$ has been adopted in Figures 18 and 20. Moreover, the vertical axes of 


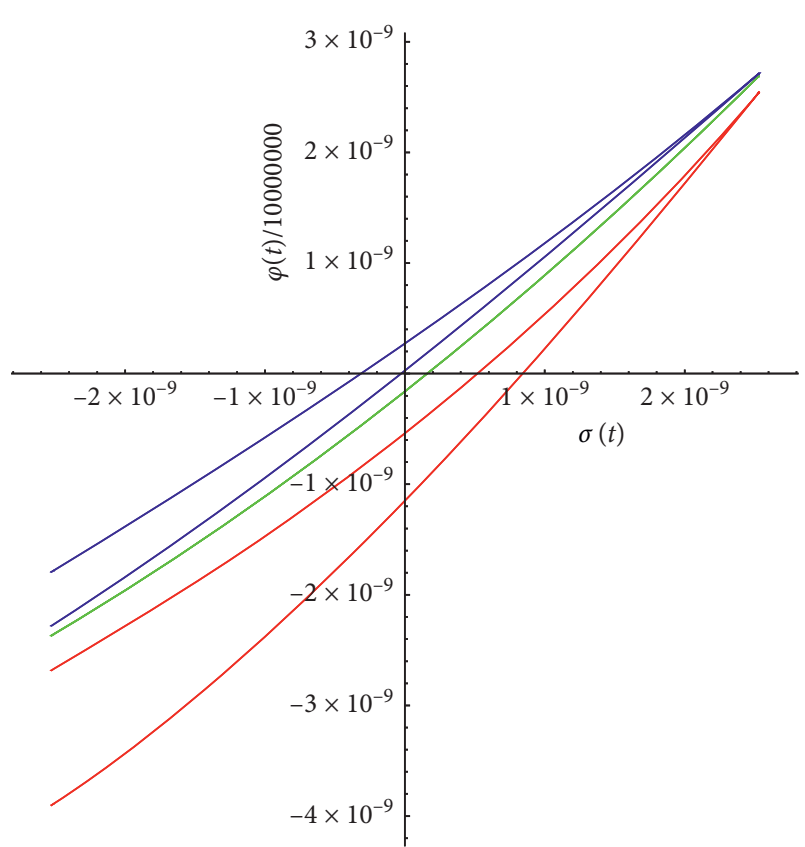

FIgURE 17: $\varphi(t)-\sigma(t)$ of the memcapacitor under the periodic waveform with $I_{m}>0: \alpha=0.75$ (red), $\alpha=1$ (green), and $\alpha=1.25$ (blue).

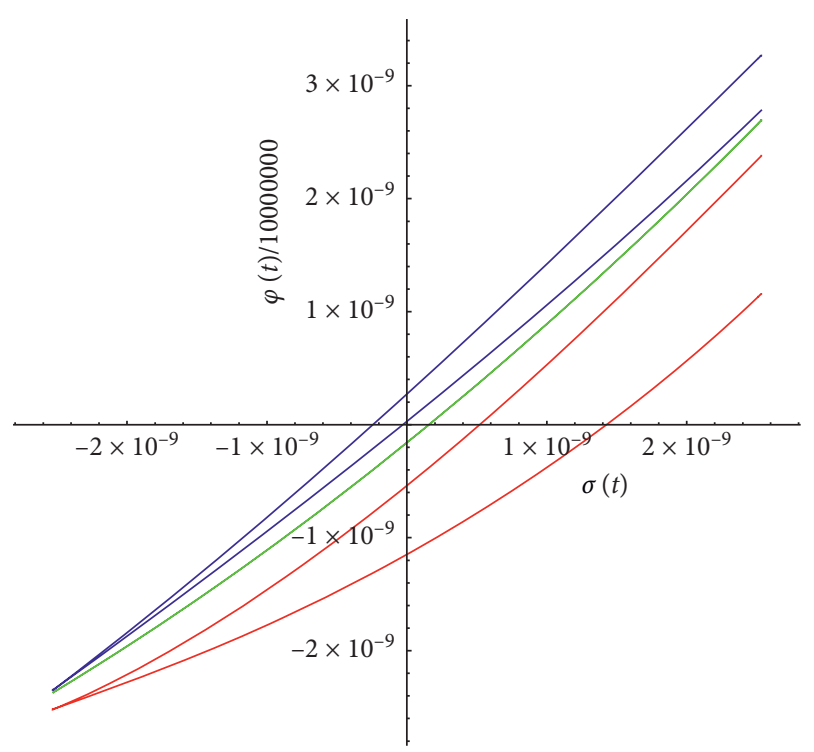

FIGURE 18: $\varphi(t)-\sigma(t)$ of the memcapacitor under the periodic waveform with $I_{m}<0: \alpha=0.75$ (red), $\alpha=1$ (green), and $\alpha=1.25$ (blue).

these figures have been scaled down by $10^{7}$ for visibility. Similarly to the meminductor, it has been found that the constitutive relation of the memcapacitor with fractionalorder kinetics becomes ambiguous unless $\alpha \neq 1$ as the oneto-one correspondence between $\varphi(t)$ and $\sigma(t)$ ceased to be existed, as shown in Figures 17 and 18. Thus, it can be stated that the fractional-order domain generalization cannot preserve the unambiguous constitutive relation which is one of the basic fingerprints of the memcapacitor [40]. However,

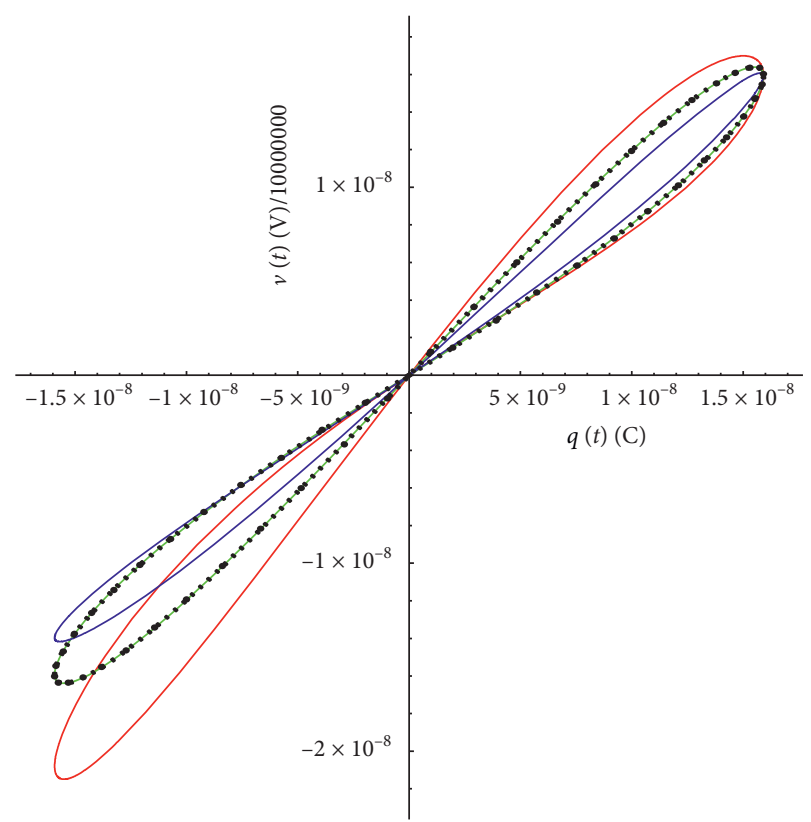

FIGURE 19: $v(t)-q(t)$ of the memcapacitor under the periodic waveform with $I_{m}>0$ : $\alpha=0.75$ (red), $\alpha=1$ (green), $\alpha=1.25$ (blue), and SPICE model (black dots).

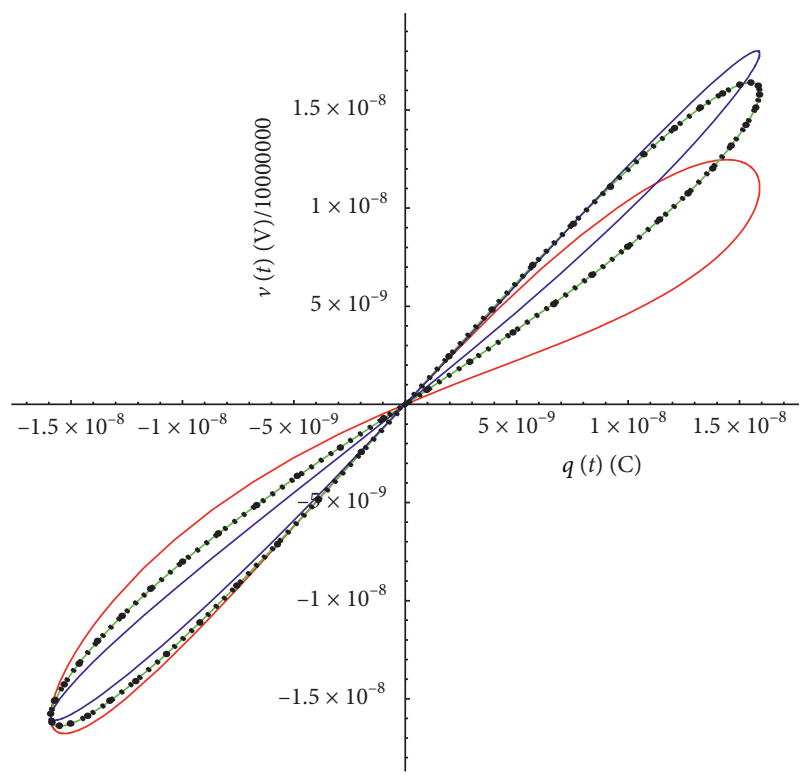

FIgURE 20: $v(t)-q(t)$ of the memcapacitor under the periodic waveform with $I_{m}<0$ : $\alpha=0.75$ (red), $\alpha=1$ (green), $\alpha=1.25$ (blue), and SPICE model (black dots).

the $2^{\text {nd }}$ basic fingerprint, i.e., the pinched hysteresis loop in the $v(t)-q(t)$ characteristic, can be preserved despite the asymmetricity when $\alpha \neq 1$, as shown in Figures 19 and 20 where the strong agreements between the $v(t)-q(t)$ characteristic with $\alpha=1$ and the SPICE memcapacitor modelbased counterpart with rectangular window function can be observed. Similarly to the meminductor, it can be seen that the lobe area of the pinched hysteresis loop of the memcapacitor with fractional-order kinetics is also inversely proportional to $\alpha$. Therefore, more memory effect and less 
linearity can also be obtained by using the memcapacitor with lower $\alpha$, and vice versa.

Unlike the meminductor, we have found that the fractional-order kinetic memcapacitor is balanced. For illustration, the areas of the upper lobe and lower lobe of the $v(t)-q(t)$ pinched hysteresis loop, i.e., $A_{\mathrm{UC}}$ and $A_{\mathrm{LC}}$, must be calculated by using the following equations:

$$
\begin{aligned}
& A_{\mathrm{UC}}=\int_{-\theta / \omega}^{(\pi-\theta) / \omega} v(t) \mathrm{d} q(t), \\
& A_{\mathrm{LC}}=\int_{(\pi-\theta) / \omega}^{(2 \pi-\theta) / \omega} v(t) \mathrm{d} q(t) .
\end{aligned}
$$

Since $v(t)=D_{M}(t) q(t)$ and $q(t)=\int_{0}^{t} i(\tau) \mathrm{d} \tau$, where $i(t)=$ $I_{m} \cos (\omega t+\theta), A_{\mathrm{UC}}$ and $A_{\mathrm{LC}}$ can be obtained by using (65)-(67) as follows:

$$
\begin{aligned}
& A_{\mathrm{UC}}=-\frac{2}{3} \frac{k_{C} I_{m}^{3}}{\omega^{\alpha+3}} \sin \left(\frac{\alpha \pi}{2}\right)\left(D_{\max }-D_{\min }\right), \\
& A_{\mathrm{LC}}=\frac{2}{3} \frac{k_{C} I_{m}^{3}}{\omega^{\alpha+3}} \sin \left(\frac{\alpha \pi}{2}\right)\left(D_{\max }-D_{\min }\right),
\end{aligned}
$$

which show that $A_{\mathrm{UC}}+A_{\mathrm{LC}}=0$. Since $A_{\mathrm{UC}}$ and $A_{\mathrm{LC}}$, respectively, refer to the energy intake and energy dissipated during the positive and negative half cycles, $A_{\mathrm{UC}}+A_{\mathrm{LC}}$ and its multiple can be interpreted in a similar manner to $A_{\mathrm{UL}}+A_{\mathrm{LL}}$ and its multiple. So, $A_{\mathrm{UC}}+A_{\mathrm{LC}}=0$ means that zero average power consumption is obtained, and therefore, the memcapacitor with fractional-order kinetics is balanced. Moreover, both $A_{\mathrm{UC}}$ and $A_{\mathrm{LC}}$ are independent of $\theta$, and so do the energy intake and energy dissipated during the positive and negative half cycles unlike those of the fractional-order kinetic meminductor.

Finally, it can be seen that the fractional-order domain generalization preserves the $3^{\text {rd }}$ basic fingerprint of the memcapacitor, i.e., identical time instants at which $v(t)$ and $q(t)$ cross zero levels. As illustrations, we simulate $v(t)$ of the fractional-order kinetic memcapacitor with various $\alpha$ 's, as depicted in Figures 21 and 22 where $q(t)$ has also been included, $v(t)$ 's have been divided by $10^{7}$ for visibility, and $\theta=0 \mathrm{rad}, D_{\min }=0.1 \mathrm{MF}^{-1}, D_{\max }=0.1 \mathrm{GF}^{-1}$, and $D(0)=$ $0.01 \mathrm{GF}^{-1}$ have been assumed. Obviously, these figures show that $v(t)$ and $q(t)$ employ identical $t_{x 0}$. Since $i(t)=$ $I_{m} \cos (\omega t+\theta), q(t)=\left(I_{m} / \omega\right) \sin (\omega t+\theta)$ as $q(t)=\int_{0}^{t} i(\tau) \mathrm{d} \tau$. Therefore, $t_{x 0}$ can be obtained as given by (59).

Finally, the effects of the nonlinear dopant drift will be analyzed. By using (27) and (31), the resulting $D_{M}(t)$ with arbitrary $p$ and $p=1$ can be, respectively, found as

$$
\begin{aligned}
& \sum_{r=0}^{\infty} \frac{\left(\left(D_{M}(t)-D_{\min }\right) /\left(D_{\max }-D_{\min }\right)\right)^{2 p r+1}-\left(\left(D_{M}(0)-D_{\min }\right) /\left(D_{\max }-D_{\min }\right)\right)^{2 p r+1}}{2 p r+1} \\
& =\frac{j k_{C} \Gamma(2-\alpha) I_{m}}{\omega^{1+\alpha}}[\exp (j \theta) \Gamma(\alpha,-j \omega t)-\exp (-j \theta) \Gamma(\alpha, j \omega t)] \\
& D_{M}(t)=D_{\min }+\left(\tanh \left[\tanh ^{-1}\left[\frac{D_{M}(0)-D_{\min }}{D_{\max }-D_{\min }}\right]+\frac{j k_{C} \Gamma(2-\alpha) I_{m}}{\omega^{1+\alpha}}[\exp (j \theta) \Gamma(\alpha,-j \omega t)-\exp (-j \theta) \Gamma(\alpha, j \omega t)]\right]\right)\left(D_{\max }-D_{\min }\right) .
\end{aligned}
$$

If $\alpha$ approaches 0 , we have

$$
\begin{gathered}
\sum_{r=0}^{\infty} \frac{\left(\left(D_{M}(t)-D_{\min }\right) /\left(D_{\max }-D_{\min }\right)\right)^{2 p r+1}-\left(\left(D_{M}(0)-D_{\min }\right) /\left(D_{\max }-D_{\min }\right)\right)^{2 p r+1}}{2 p r+1}=\frac{2 k_{C} \Gamma(2-\alpha) I_{m}}{\omega}[c i(\omega t) \sin (\theta)+s i(\omega t) \cos (\theta)] \\
D_{M}(t)=D_{\min }+\left(\tanh \left[\tanh ^{-1}\left[\frac{D_{M}(0)-D_{\min }}{D_{\max }-D_{\min }}\right]+\frac{2 k_{C} \Gamma(2-\alpha) I_{m}}{\omega}[c i(\omega t) \sin (\theta)+s i(\omega t) \cos (\theta)]\right]\right)\left(D_{\max }-D_{\min }\right),
\end{gathered}
$$

as $\int_{0}^{t} \tau^{\alpha-1} z(\tau) \mathrm{d} \tau$ approaches $\int_{0}^{t} \tau^{-1} z(\tau) \mathrm{d} \tau$. 


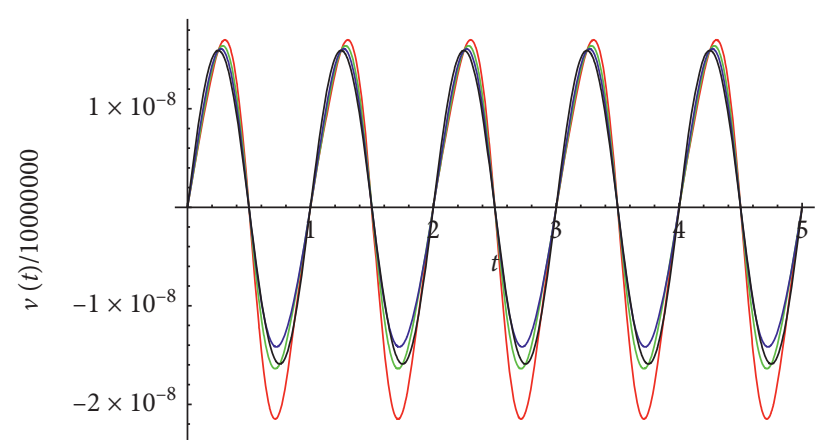

Figure 21: $v(t)$ of the memcapacitor under the periodic waveform with $I_{m}>0: \alpha=0.75$ (red), $\alpha=1$ (green), $\alpha=1.25$ (blue), and $q(t)$ (black).

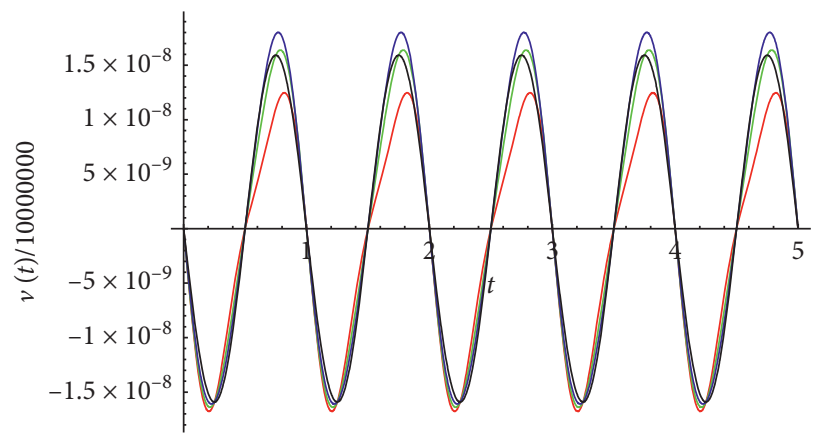

FIgURe 22: $v(t)$ of the memcapacitor under the periodic waveform with $I_{m}<0: \alpha=0.75$ (red), $\alpha=1$ (green), $\alpha=1.25$ (blue), and $q(t)$ (black).

But if $\alpha$ approaches 1 instead, we have

$$
\begin{aligned}
& \sum_{r=0}^{\infty} \frac{\left(\left(D_{M}(t)-D_{\min }\right) /\left(D_{\max }-D_{\min }\right)\right)^{2 p r+1}-\left(\left(D_{M}(0)-D_{\min }\right) /\left(D_{\max }-D_{\min }\right)\right)^{2 p r+1}}{2 p r+1} \\
& =-\frac{2 k_{C} \Gamma(2-\alpha) I_{m} \cos (\omega t+\theta)}{\omega^{2}}, \\
& D_{M}(t)=D_{\text {min }}+\left(\tanh \left[\tanh ^{-1}\left[\frac{D_{M}(0)-D_{\min }}{D_{\max }-D_{\min }}\right]-\frac{2 k_{C} \Gamma(2-\alpha) I_{m} \cos (\omega t+\theta)}{\omega^{2}}\right]\right)\left(D_{\max }-D_{\min }\right) .
\end{aligned}
$$

This is because $\int_{0}^{t} \tau^{\alpha-1} z(\tau) \mathrm{d} \tau$ approaches $\int_{0}^{t} z(\tau) \mathrm{d} \tau$.

By keeping in mind that $C_{M}(t)=1 / D_{M}(t)$ and applying the above equations, we can simulate $C_{M}(t)$ affected by the nonlinear dopant drift as dashed curved in Figures 23 and 24 where those based on the linear dopant drift have been included as normal curves for comparison. We also simulate the $v(t)-q(t)$ characteristic, as shown in Figures 25 and 26 where the dashed curves have been used for being distinguished from the linear dopant drift-based $v(t)-q(t)$ depicted in Figures 19 and 20. Here, we choose $p=1$ where $\theta=0 \mathrm{rad}, D_{\min }=0.1 \mathrm{MF}^{-1}, D_{\max }=0.1 \mathrm{GF}^{-1}$, and $D(0)=0.01 \mathrm{GF}^{-1}$ have also been assumed for ceteris paribus.

From Figures 23-26, where strong agreements between our results with the nonlinear dopant drift and those based on the SPICE model with Jocklecar's window function and $p=1$ can be observed, and Figures 19 and 20 , it can be seen that the nonlinear dopant drift reduces the effects of $\alpha$ on the variation in $C_{M}(t)$ and variation in nonlinearity of the device. Therefore, the memcapacitor with the nonlinear dopant drift is more robust to the effects of fractional kinetic transport. Similarly to the meminductor, the memcapacitor with the nonlinear dopant drift also becomes more nonlinear with increasing $\alpha$ and employs a more symmetric pinched hysteresis loop than that with the linear dopant drift.

\section{The Emulation by Using Memristor}

Despite employing the fractional-order kinetics, the constitutive relations of the meminductor and memcapacitor remain as given by (1) and (4). As a result, the emulation of memreactance by using the memristor and conventional memristor to meminductor/memcapacitor emulators $[33,46]$ remains possible. In this section, such possibility will be illustrated.

Since the constitutive relation of the memristor can be given by $[5,7]$ 


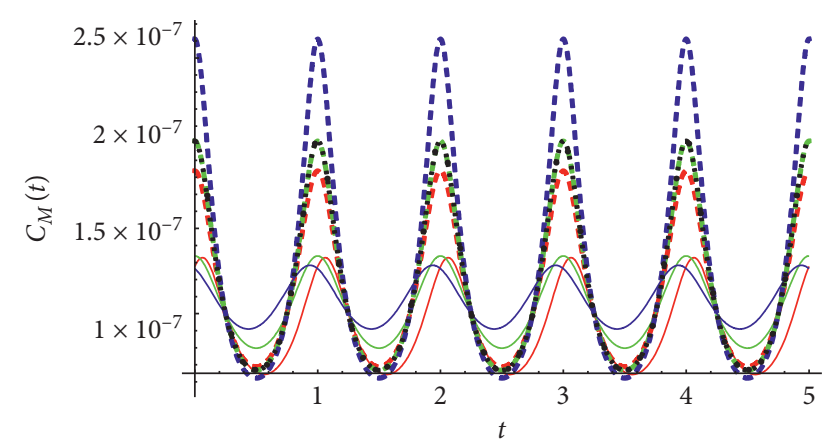

FIgURE 23: $C_{M}(t)$ of the memcapacitor with linear (normal curve) and nonlinear (dashed curve) dopant drifts under the periodic waveform with $I_{m}>0$ : $\alpha=0.75$ (red), $\alpha=1$ (green), $\alpha=1.25$ (blue), and SPICE model (black dots).

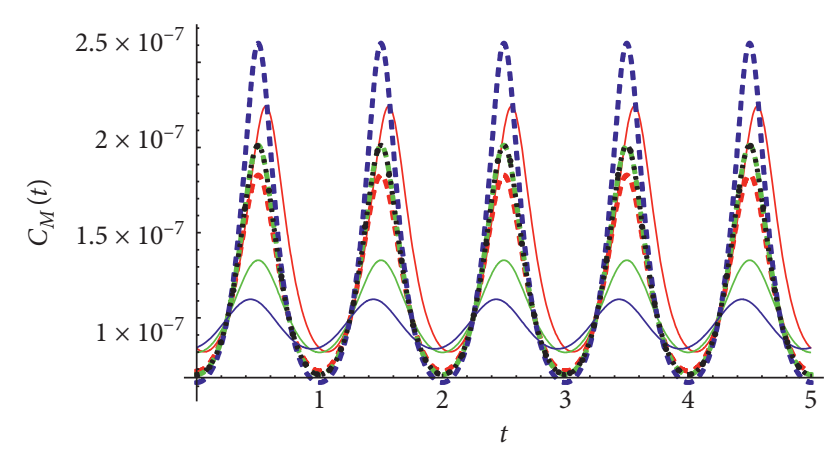

FIgURe 24: $C_{M}(t)$ of the memcapacitor with linear (normal curve) and nonlinear (dashed curve) dopant drifts under the periodic waveform with $I_{m}<0$ : $\alpha=0.75$ (red), $\alpha=1$ (green), $\alpha=1.25$ (blue), and SPICE model (black dots).

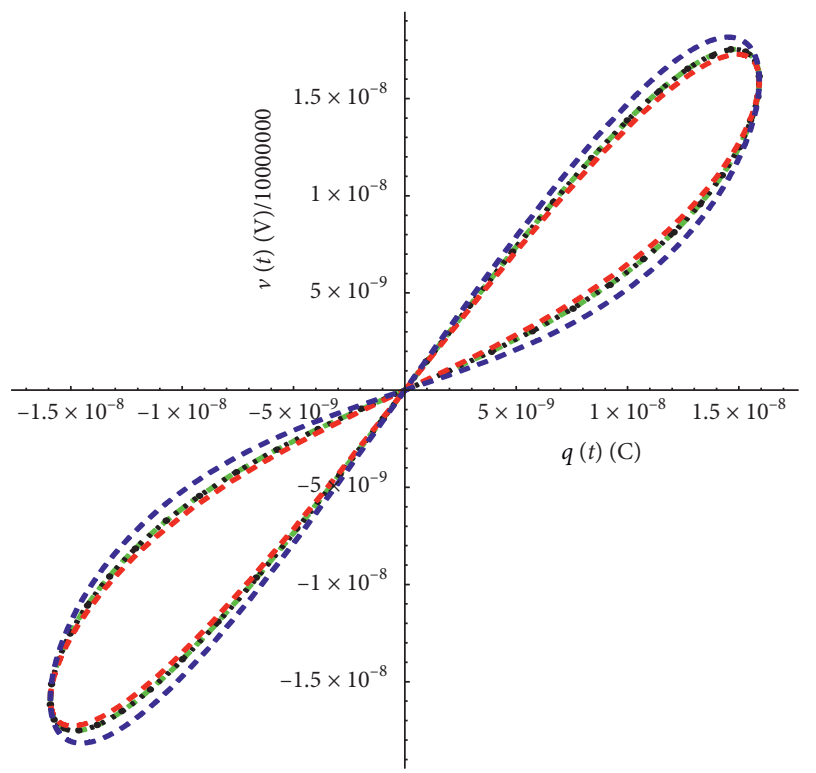

FIGURE 25: $v(t)-q(t)$ of the memcapacitor with the nonlinear dopant drift under the periodic waveform with $I_{m}>0: \alpha=0.75$ (red), $\alpha=1$ (green), $\alpha=1.25$ (blue), and SPICE model (black dots).

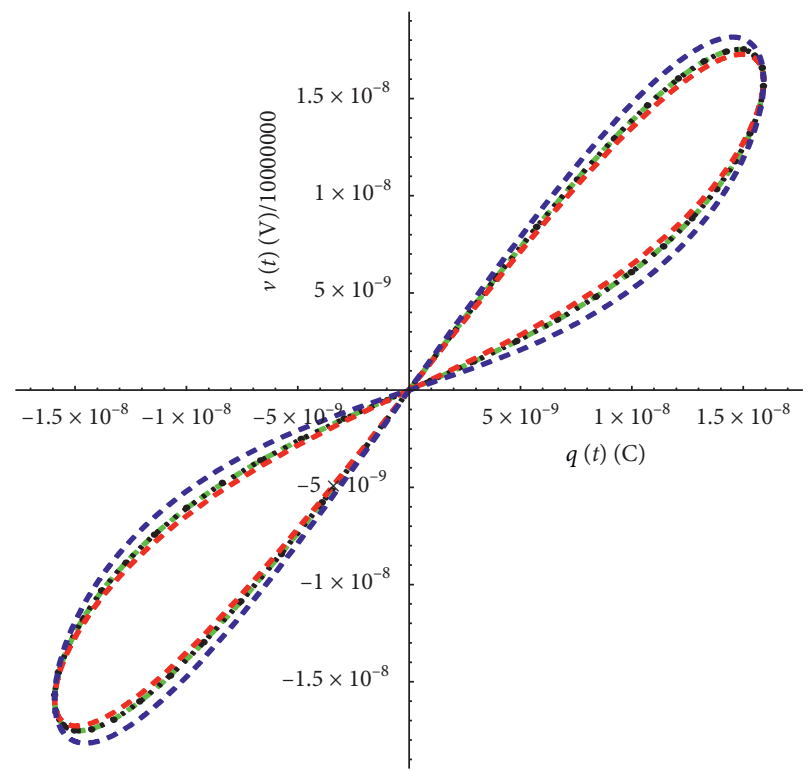

FIGURE 26: $v(t)-q(t)$ of the memcapacitor with the nonlinear dopant drift under the periodic waveform with $I_{m}>0: \alpha=0.75$ (red), $\alpha=1$ (green), $\alpha=1.25$ (blue), and SPICE model (black dots).

$$
M(t)=\frac{\mathrm{d} \varphi(t)}{\mathrm{d} q(t)},
$$

(1) can be obtained via the following transformation:

$$
\begin{aligned}
& \rho_{L}(t)=a \varphi_{M}(t), \\
& q_{L}(t)=b q_{M}(t),
\end{aligned}
$$

where $a$ and $b$ are real constants. Moreover, the subscripts $L$ and $M$ refer to the meminductor and memristor, respectively.

By taking differentiation with respect to $t$ for both sides of the above transformation equations, we have

$$
\begin{gathered}
\varphi_{L}(t)=a v_{M}(t), \\
i_{L}(t)=b i_{M}(t) .
\end{gathered}
$$

As $\varphi_{L}(t)=\int_{0}^{t} v_{L}(\tau) \mathrm{d} \tau$, the following transformation equations can be obtained:

$$
\begin{aligned}
& v_{L}(t)=a \frac{\mathrm{d}}{\mathrm{d} t} v_{M}(t), \\
& i_{L}(t)=b i_{M}(t) .
\end{aligned}
$$

Since we assume that $i_{L}(t)$ and $i_{M}(t)$ flow in the opposite directions, $i_{L}(t)=b i_{M}(t)$ becomes $i_{L}(t)=-b i_{M}(t)$. After taking the Laplace transformation for both sides of the equations, the following linear transformation that transforms the memristor to the meminductor can be obtained:

$$
\left[\begin{array}{c}
V_{L}(s) \\
I_{L}(s)
\end{array}\right]=\left[\begin{array}{cc}
a s & 0 \\
0 & b
\end{array}\right]\left[\begin{array}{c}
V_{M}(s) \\
-I_{M}(s)
\end{array}\right] .
$$

By using (76), $L_{M}(t)$ can be given in terms of $M(t)$ as follows: 


$$
L_{M}(t)=\frac{a}{b} M(t)
$$

For obtaining $L_{M}(t)$ exactly as given by (2) and the fractional-order kinetics, the following $M(t)$ must be used:

$$
M(t)=\frac{b}{a}\left(\sqrt{L_{\min }}+x_{M}(t)\left(\sqrt{L_{\max }}-\sqrt{L_{\min }}\right)\right),
$$

where $x_{M}(t)$ can be mathematically defined by (19) with $x(t)=x_{M}(t), z(t)=i(t)$, and $k=k_{M}$. For the meminductor emulation, $k_{M}=k_{L}$ must be satisfied.

At this point, it can be seen that the meminductor can be emulated by using the memristor and the conventional memristor to the meminductor emulator [33] despite taking the fractional-order kinetics into account. In addition, the kinetics of the memristor must be of fractional order in order to obtain the meminductor employing such fractional-order kinetics. Note also that Jocklecar's window function has been adopted for modelling the nonlinear dopant drift of the memristor for ceteris paribus.

Now, the emulation of the memcapacitor with fractional-order kinetics by using the memristor will be discussed. It can be seen that (4) can be obtained by applying the following transformation to (72):

$$
\begin{aligned}
& \varphi_{C}(t)=b \varphi_{M}(t), \\
& \sigma_{C}(t)=a q_{M}(\mathrm{t}),
\end{aligned}
$$

where the subscript $C$ refers to the memcapacitor.

After the differentiation with respect to $t$ and rearranging by keeping the relationship between charge and current in mind, we have

$$
\begin{aligned}
& v_{C}(t)=b v_{M}(t), \\
& i_{C}(t)=-a \frac{\mathrm{d}}{\mathrm{d} t} i_{M}(t) .
\end{aligned}
$$

Note that the opposite directions of $i_{C}(t)$ and $i_{M}(t)$ have been assumed.

By applying the Laplace transformation, the following linear transformation that transforms the memristor to the memcapacitor can be obtained:

$$
\left[\begin{array}{c}
V_{C}(s) \\
I_{C}(s)
\end{array}\right]=\left[\begin{array}{cc}
b & 0 \\
0 & a s
\end{array}\right]\left[\begin{array}{c}
V_{M}(s) \\
-I_{M}(s)
\end{array}\right],
$$

which implies that $D_{M}(t)$ can be given in terms of $M(t)$ as follows:

$$
D_{M}(t)=\left(\frac{b}{a} M(t)\right)^{-1} .
$$

In order to obtain $D_{M}(t)$ exactly as given by (5), $M(t)$ must be given by

$$
M(t)=\frac{a}{b}\left(D_{\min }+x_{M}(t)\left(D_{\max }-D_{\min }\right)\right)^{-1} .
$$

Note that $x_{M}(t)$ can be defined similarly to that of (78) as Jocklecar's window function has been assumed for ceteris paribus where $k_{M}=k_{C}$ must be satisfied. At this point, it can be seen that the memcapacitor can be emulated by using the memristor and the conventional memristor to the meminductor emulator [47] despite taking the fractional-order kinetics into account. The kinetics of the memristor must be of fractional order for obtaining the memcapacitor with fractional-order kinetics.

\section{The Influence of Fractional-Order Kinetics on Memreactance-Based Circuits}

For studying the influence of the fractional-order kinetics on the meminductor-based circuit, the meminductor-based oscillator [36] depicted here in Figure 27 has been chosen. By taking the fractional-order kinetics of the meminductor into account, the dynamical equation of this circuit can be given as follows:

$$
\begin{aligned}
\frac{\mathrm{d} u_{1}(t)}{\mathrm{d} t} & =A \varphi(t)+B \varphi(t) \rho_{\alpha}(t)-C u_{1}(t), \\
\frac{\mathrm{d} u_{2}(t)}{\mathrm{d} t} & =D u_{2}(t)-E \varphi(t)-F \varphi(t) \rho_{\alpha}(t), \\
\frac{\mathrm{d} \varphi(t)}{\mathrm{d} t} & =u_{2}(t)-u_{1}(t), \\
D^{\alpha}\left[\rho_{\alpha}(t)\right] & =\varphi(t),
\end{aligned}
$$

where $\quad A=\left(L_{\max } / C_{1}\right), \quad B=\left(k\left(L_{\min }-L_{\max }\right) / C_{1}\right)$, $C=\left(1 / C_{1} R\right), \quad D=\left(G / C_{2}\right), \quad E=\left(L_{\max } / C_{2}\right), \quad$ and $F=\left(k\left(L_{\min }-L_{\max }\right) / C_{2}\right)$. Note also that the linear dopant drift model has been assumed in the formulation of (84) in which the voltage terms have been denoted by $u_{1}(t)$ and $u_{2}(t)$, for ceteris paribus as these model and voltage symbols have been assumed in [36].

According to [36], this meminductor-based oscillator with integer kinetics generates the periodic output if we let $A=1, B=6, C=4.6, D=0.4, E=4$, and $F=1$ and becomes chaotic when $D=0.7$ and other parameters remain the same. By using (77) with $\left\{A, B, C, D, E, F, u_{1}(0), u_{2}(0)\right.$, $\left.\varphi(0), \rho_{\alpha}(0)\right\}=\{1,6,4.6,0.4,4,1,2.5,0.01,0.01,0.01\}$ and $\{A$, $\left.B, C, D, E, F, u_{1}(0), u_{2}(0), \varphi(0), \rho_{\alpha}(0)\right\}=\{1,6,4.6,0.7,4,1$, $0.01,0.01,0.01,0.01\}$ [28], we can simulate $u_{2}(t)$ with various a's, as depicted in Figures 28-30 and Figures 31-33. These figures show that $u_{2}(t)$ remains periodic and chaotic when $\left\{A, B, C, D, E, F, u_{1}(0), u_{2}(0), \varphi(0), \rho_{\alpha}(0)\right\}=\{1,6,4.6,0.4,4,1$, $2.5,0.01,0.01,0.01\}$ and $\left\{A, B, C, D, E, F, u_{1}(0), u_{2}(0), \varphi(0)\right.$, $\left.\rho_{\alpha}(0)\right\}=\{1,6,4.6,0.7,4,1,0.01,0.01,0.01,0.01\}$, respectively, despite the incorporation of the meminductor's fractionalorder kinetics. Note that the strong agreements between $u_{2}(t)$ with $\alpha=1$ and that based on the conventional integer kinetics which can be obtained by allowing all derivatives to be of the conventional type [36] can be observed. It can also be seen that $u_{2}(t)$ becomes more oscillatory with the increasing $\alpha$. Therefore, it can be stated that incorporating the fractional-order kinetics can offer more degrees of freedom as the amount of oscillation becomes now controllable via $\alpha$.

In addition, it can be seen from these simulation results that $D$ is the most prominent control parameter of this circuit because it solely determines whether the circuit is periodic or chaotic. So, it is worthy to further explore the 


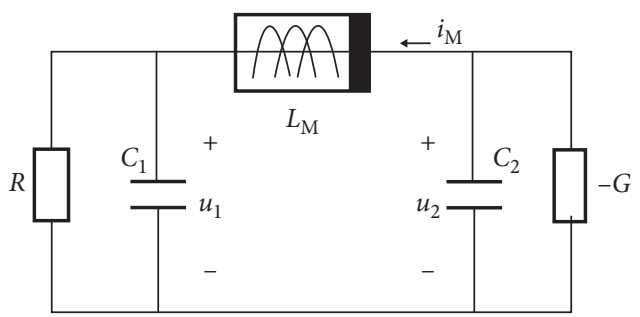

FIGURE 27: The candidate meminductor-based oscillator [28].

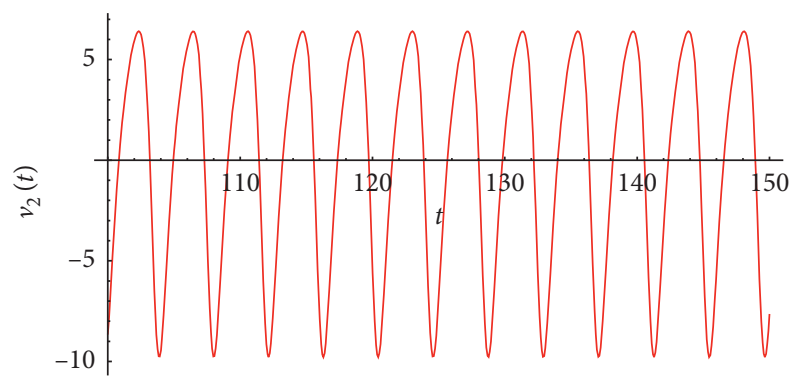

Figure 28: $u_{2}(t)$ when $\left\{A, B, C, D, E, F, u_{1}(0), u_{2}(0), \varphi(0), \rho_{\alpha}(0)\right\}=$ $\{1,6,4.6,0.4,4,1,2.5,0.01,0.01,0.01\}: \alpha=0.95$.

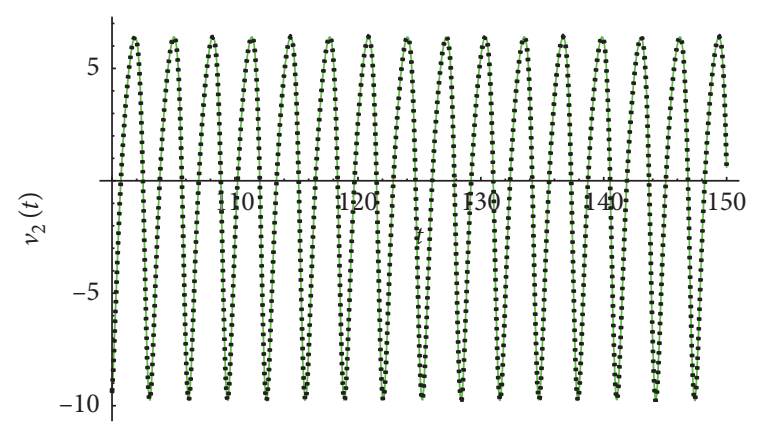

Figure 29: $u_{2}(t)$ when $\left\{A, B, C, D, E, F, u_{1}(0), u_{2}(0), \varphi(0), \rho_{\alpha}(0)\right\}=$ $\{1,6,4.6,0.4,4,1,2.5,0.01,0.01,0.01\}: \alpha=1$ and integer kinetics (dotted line).

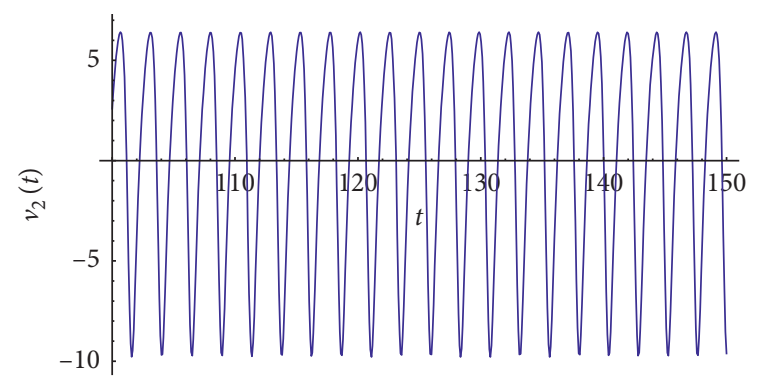

Figure 30: $u_{2}(t)$ when $\left\{A, B, C, D, E, F, u_{1}(0), u_{2}(0), \varphi(0), \rho_{\alpha}(0)\right\}=$ $\{1,6,4.6,0.4,4,1,2.5,0.01,0.01,0.01\}: \alpha=1.05$.

effect of $D$ on the circuit's dynamics. In order to do so, we simulate the $3 \mathrm{D}$ phase portraits of $u_{1}(t), u_{2}(t)$, and $\varphi(t)$ by using (77). It should be mentioned here that $\rho_{\alpha}(t)$ is not of our interest because it is merely a fractional integral of $\varphi(t)$ as can be seen from (77). Here, we assume $\{A, B, C, \alpha, E, F$,

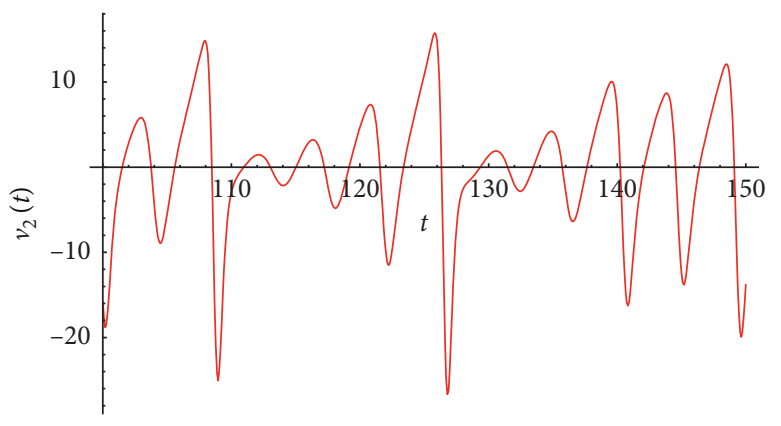

Figure 31: $u_{2}(t)$ when $\left\{A, B, C, D, E, F, u_{1}(0), u_{2}(0), \varphi(0), \rho_{\alpha}(0)\right\}=$ $\{1,6,4.6,0.7,4,1,0.01,0.01,0.01,0.01\}: \alpha=0.95$.

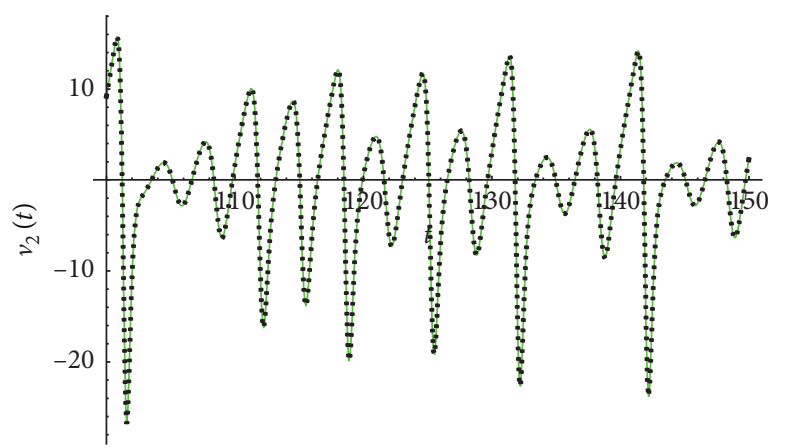

Figure 32: $u_{2}(t)$ when $\left\{A, B, C, D, E, F, u_{1}(0), u_{2}(0), \varphi(0), \rho_{\alpha}(0)\right\}=$ $\{1,6,4.6,0.7,4,1,0.01,0.01,0.01,0.01\}: \alpha=1$ and integer kinetics (dotted line).

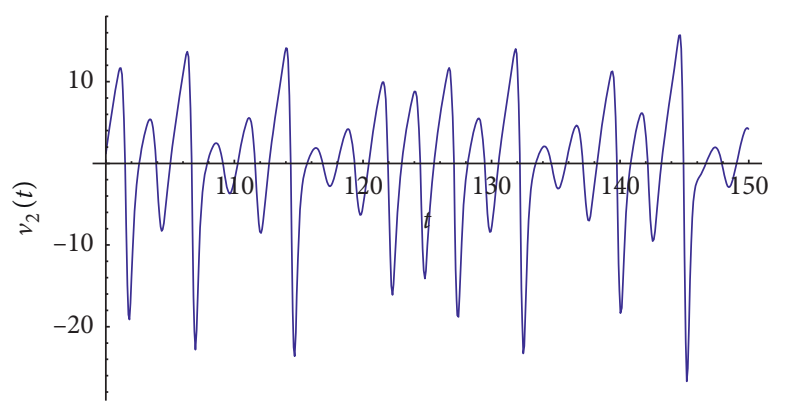

Figure 33: $u_{2}(t)$ when $\left\{A, B, C, D, E, F, u_{1}(0), u_{2}(0), \varphi(0), \rho_{\alpha}(0)\right\}=$ $\{1,6,4.6,0.7,4,1,0.01,0.01,0.01,0.01\}: \alpha=1.05$.

$\left.u_{1}(0), u_{2}(0), \varphi(0), \rho_{\alpha}(0)\right\}=\{1,6,4.6,0.95,4,1,2.5,0.01,0.01$, 0.01 \} and let $D$ be the bifurcation parameter. As a result, the following phase portraits can be obtained.

From Figure 34 in which $D=0.7$ has been assumed, a chaotic attractor can be observed, thus confirming the above simulation result depicted in Figure 31. In addition, we have also found by using the numerical computation with MATHEMATICA that the corresponding Lyapunov exponents are given by $\mathrm{LE}_{1}=0.152939, \mathrm{LE}_{2}=0.0294933$, $\mathrm{LE}_{3}=-0.00451014$, and $\mathrm{LE}_{4}=-4.00792$ where the Lyapunov dimension can be found as $D_{L}=3.0463$. From the Lyapunov exponents, it can be seen that a contracting volume with expansions in two directions in the phase space of the 


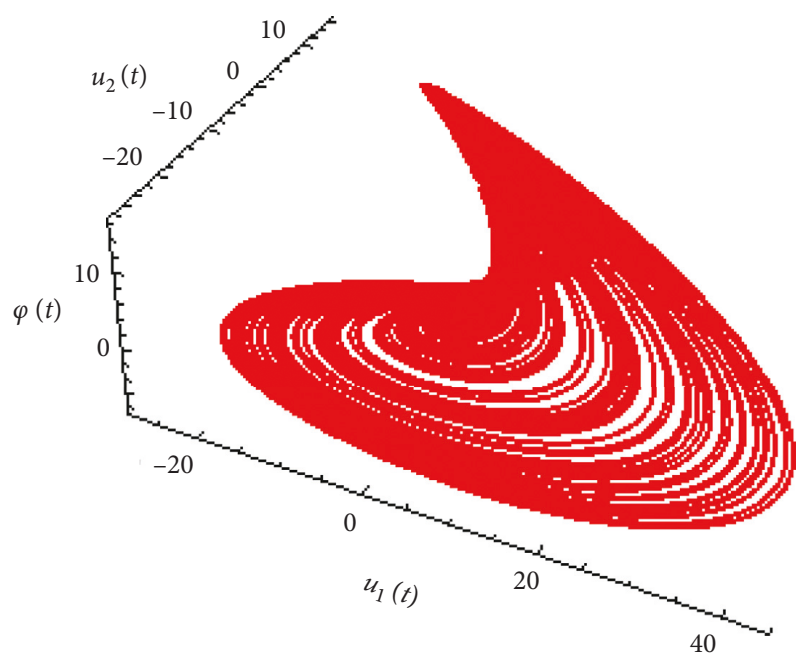

FIGURE 34: 3D phase portraits of $u_{1}(t), u_{2}(t)$, and $\varphi(t)$ when $\{A, B, C$, $\left.\alpha, E, F, u_{1}(0), u_{2}(0), \varphi(0), \rho_{\alpha}(0)\right\}=\{1,6,4.6,0.95,4,1,0.01,0.01,0.01$, $0.01\}: D=0.7$.

attractor indicates that the chaotic dissipative behavior can be observed because $\mathrm{LE}_{1}+\mathrm{LE}_{2}+\mathrm{LE}_{3}+\mathrm{LE}_{4}<0$ where $\mathrm{LE}_{1}>0$ and $L_{2}>0$. In addition, the obtained fractional number $D_{L}$ states that the manifold in the phase space is a strange attractor which also indicates the chaotic behavior. From Figures 35-38, it can be seen that the circuit becomes marginally and asymptotically stable by reducing $D$ where the asymptotic stability can be obtained if $D \leq 0.1$ has been satisfied due to the observed stable focus.

For a similar study on the memcapacitor-based circuit, the memcapacitor-based synaptic network [37] which is depicted here in Figure 39 will be considered. If a current pulse has been applied as an input to this circuit, the resulting synaptic weight, $\psi(t)$, will be given by [38]

$$
\psi(t)=\frac{D_{a}(t)-D_{b}(t)}{D_{a}(t)+D_{b}(t)},
$$

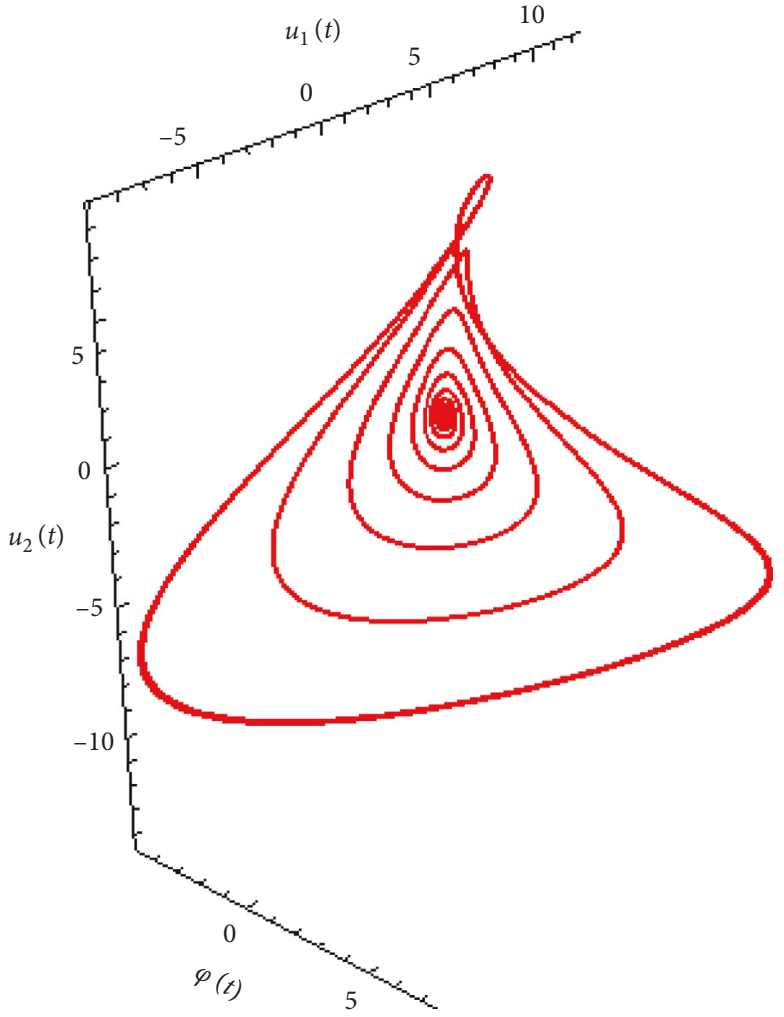

Figure 35: 3D phase portraits of $u_{1}(t), u_{2}(t)$, and $\varphi(t)$ when $\{A, B, C$, $\left.\alpha, E, F, u_{1}(0), u_{2}(0), \varphi(0), \rho_{\alpha}(0)\right\}=\{1,6,4.6,0.95,4,1,0.01,0.01,0.01$, $0.01\}: D=0.5$.

where $D_{a}(t)=D_{m 2}(t)=D_{m 3}(t)$ and $D_{b}(t)=D_{m 1}(t)=D_{m 4}(t)$.

Since we take the fractional-order kinetics of the memcapacitor into account, $D_{a}(t)$ and $D_{b}(t)$ can be derived by using (45) which assumes $p=1$, for ceteris paribus as $[37,38]$ assumed such a value of $p$ in their simulations, and keeping the antiseries connection of the devices in mind as

$$
\begin{aligned}
& D_{a}(t)=D_{\min }+\tanh \left[\tanh ^{-1}\left[\frac{D_{a}(0)-D_{\min }}{D_{\max }-D_{\min }}\right]+\frac{k_{C} \Gamma(2-\alpha) I_{0} t^{\alpha+1}}{\alpha+1}\right]\left(D_{\max }-D_{\min }\right), \\
& D_{b}(t)=D_{\min }+\tanh \left[\tanh ^{-1}\left[\frac{D_{b}(0)-D_{\min }}{D_{\max }-D_{\min }}\right]-\frac{k_{C} \Gamma(2-\alpha) I_{0} t^{\alpha+1}}{\alpha+1}\right]\left(D_{\max }-D_{\min }\right),
\end{aligned}
$$

where $I_{0}$ stands for the magnitude of the applied current pulse.
Therefore, $\psi(t)$ with the fractional-order kinetics can be obtained as follows:

$\psi(t)=\frac{\left\{\tanh \left[\tanh ^{-1}\left[\left(D_{a}(0)-D_{\min }\right) /\left(D_{\max }-D_{\min }\right)\right]+\left(k_{C} \Gamma(2-\alpha) I_{0} t^{\alpha+1} /(\alpha+1)\right)\right]-\tanh \left[\tanh ^{-1}\left[\left(D_{\max }-D_{a}(0)\right) /\left(D_{\max }-D_{\min }\right)\right]-\left(k_{C} \Gamma(2-\alpha) I_{0} t^{\alpha+1} /(\alpha+1)\right)\right]\right\}\left(D_{\max }-D_{\min }\right)}{2 D_{\min }+\left\{\tanh \left[\tanh ^{-1}\left[\left(D_{a}(0)-D_{\min }\right) /\left(D_{\max }-D_{\min }\right)\right]+\left(k_{C} \Gamma(2-\alpha) I_{0} t^{\alpha+1} /(\alpha+1)\right)\right]+\tanh \left[\tanh ^{-1}\left[\left(D_{\max }-D_{a}(0)\right) /\left(D_{\max }-D_{\min }\right)\right]-\left(k_{C} \Gamma(2-\alpha) I_{0} t^{\alpha+1 /} /(\alpha+1)\right)\right]\right\}\left(D_{\max }-D_{\min }\right)}$. 


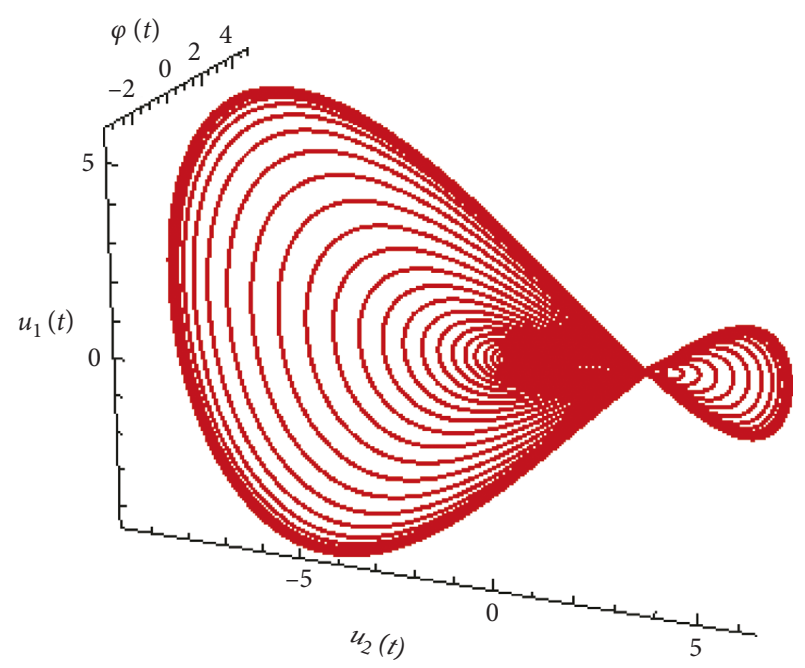

FIGURE 36: 3D phase portraits of $u_{1}(t), u_{2}(t)$, and $\varphi(t)$ when $\{A, B, C$, $\left.\alpha, E, F, u_{1}(0), u_{2}(0), \varphi(0), \rho_{\alpha}(0)\right\}=\{1,6,4.6,0.95,4,1,0.01,0.01,0.01$, $0.01\}: D=0.3$.

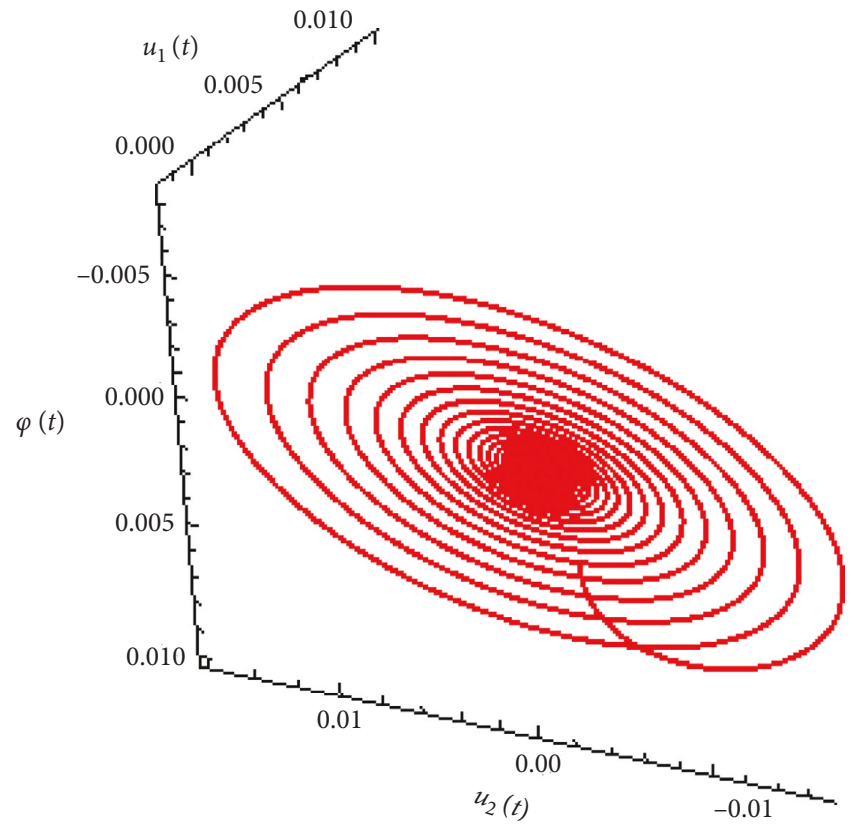

Figure 37: 3D phase portraits of $u_{1}(t), u_{2}(t)$, and $\varphi(t)$ when $\{A, B, C$, $\left.\alpha, E, F, u_{1}(0), u_{2}(0), \varphi(0), \rho_{\alpha}(0)\right\}=\{1,6,4.6,0.95,4,1,0.01,0.01,0.01$, $0.01\}: D=0.1$.

By allowing $D_{m 2}(0)=D_{m 3}(0)=0.009901 \mathrm{MF}^{-1}$ and other parameters to be similar to those of [30], $\psi(t)$ can be simulated under the assumption that $I_{0}=1 \mu \mathrm{A}$ and the current pulse duration is $2 \mathrm{sec}[37,38]$, as depicted in Figure 40. From this figure, a strong agreement between $\psi(t)$ with $\alpha=1$ and that with integer kinetics can be observed. It can also be seen that the circuit quality can be improved with increasing $\alpha$ as faster weight adjustment and more proportion of linear regions [37] can be obtained. This marks the circuit quality improvement of employing the fractionalorder kinetics.

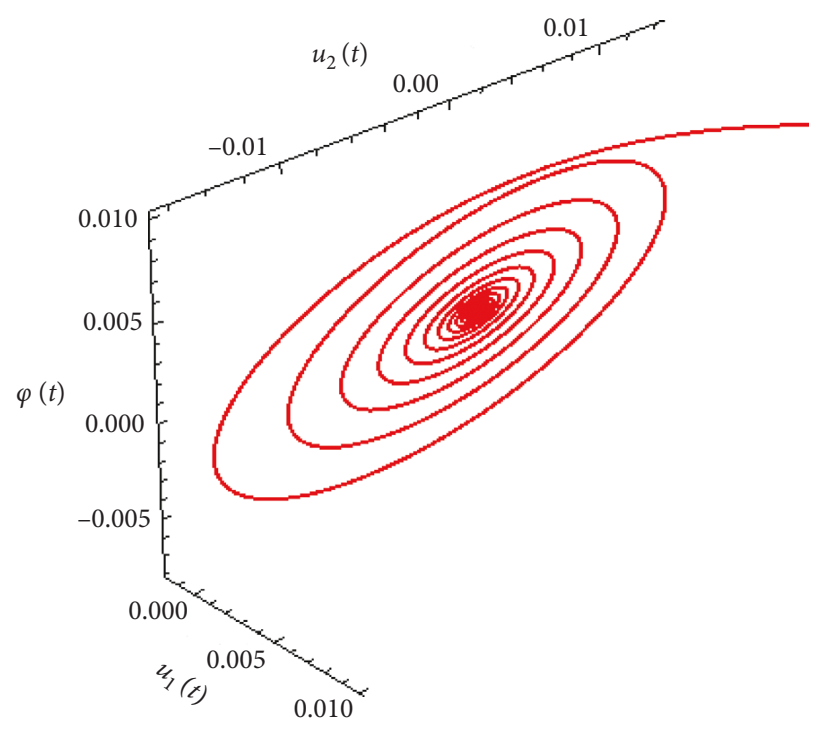

FIGURE 38: 3D phase portraits of $u_{1}(t), u_{2}(t)$, and $\varphi(t)$ when $\{A, B, C$, $\left.\alpha, E, F, u_{1}(0), u_{2}(0), \varphi(0), \rho_{\alpha}(0)\right\}=\{1,6,4.6,0.95,4,1,0.01,0.01,0.01$, $0.01\}: D=0$.

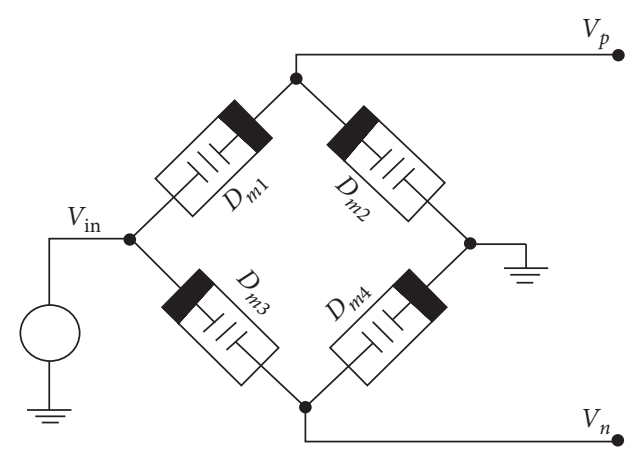

FIGURE 39: Memcapacitor-based synaptic network [37].

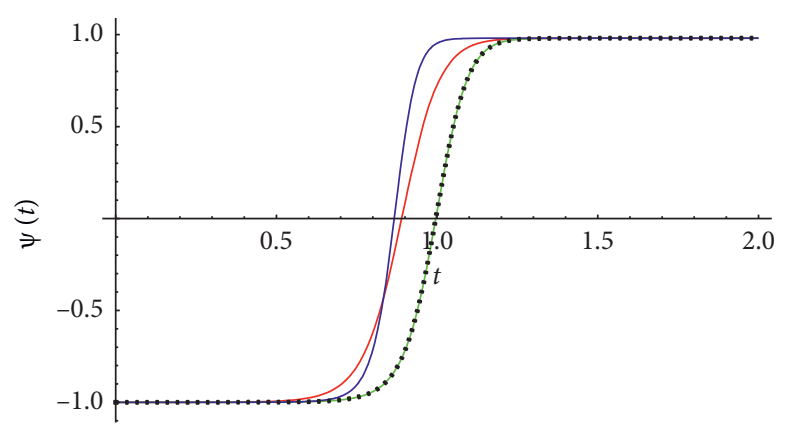

FIGURE 40: $\psi(t)\left(D_{m 2}(0)=D_{m 3}(0)=0.009901 \mathrm{MF}^{-1}\right): \alpha=0.5(\mathrm{red})$, $\alpha=1$ (green), $\alpha=1.5$ (blue), and integer kinetics (black dotted line).

Now, we let $D_{m 2}(0)=D_{m 3}(0)=0.005 \mathrm{MF}^{-1}$. As a result, $\psi(t)$ can be simulated, as depicted in Figure 41 where a strong agreement between $\psi(t)$ with $\alpha=1$ and that with integer kinetics can also be observed. From this figure, it can be seen that the circuit quality can also be improved by decreasing $D_{m 2}(0)$ and $D_{m 3}(0)$ as the faster weight adjustment can be obtained. 


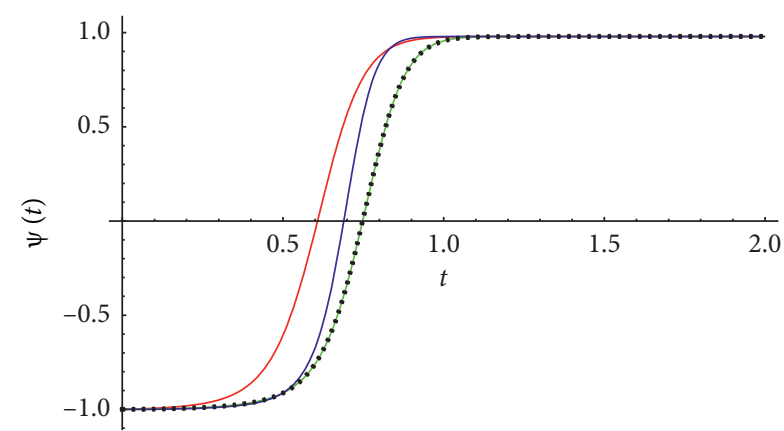

Figure 41: $\psi(t)\left(D_{m 2}(0)=D_{m 3}(0)=0.005 \mathrm{MF}^{-1}\right): \alpha=0.5$ (red), $\alpha=1$ (green), $\alpha=1.5$ (blue), and integer kinetics (black dotted line).

\section{Extension to the Fractional- Order Memreactance}

Fractional-order memreactance or memfractance in short is a common term for both the fractional-order meminductor and the fractional-order memcapacitor. According to [30], the characteristic of the fractional-order meminductor is in between those of the meminductor and memristor, whereas that of the fractional-order memcapacitor is in between those of the memristor and memcapacitor as the results of applying the concept of fractional calculus to the port equations of both the meminductor and the memcapacitor. For illustrating this issue, consider the following voltagecurrent relationship of the meminductor:

$$
v(t)=\frac{\mathrm{d}}{\mathrm{d} t} L_{M}(t) i(t) .
$$

By applying the fractional calculus concept, we have

$$
v(t)=D^{\gamma}\left[L_{M}(t) i(t)\right]
$$

which is equivalent to

$$
D^{-\gamma}[v(t)]=L_{M}(t) i(t)
$$

where $0 \leq \gamma \leq 1$.

Since $v(t)=(\mathrm{d} / \mathrm{d} t) \varphi(t)$, the following port equation of the fractional-order meminductor can be finally obtained:

$$
D^{1-\gamma}[\varphi(t)]=L_{M}(t) i(t) \text {. }
$$

By keeping in mind that the memcapacitor and meminductor are dual elements and thus so do their fractionalorder counterparts, we obtain the following port equation of the fractional-order memcapacitor by inspecting (92):

$$
D^{1-\gamma}[q(t)]=C_{M}(t) v(t) .
$$

Obviously, if $\gamma=0,(92)$ and (93) become

$$
\begin{aligned}
& v(t)=L_{M}(t) i(t), \\
& i(t)=C_{M}(t) v(t),
\end{aligned}
$$

which show that both the fractional-order meminductor and the fractional-order memcapacitor, respectively, behave in a similar manner to a memristor with memristance of $L_{M}(t)$ and memductance of $C_{M}(t)$ if $\gamma=0$.

On the contrary, if $\gamma=1,(92)$ and (93) become

$$
\begin{aligned}
& \varphi(t)=L_{M}(t) i(t), \\
& q(t)=C_{M}(t) v(t),
\end{aligned}
$$

which are the port equations of the meminductor and memcapacitor, respectively. Therefore, it can be seen that the fractional-order meminductor and fractional-order memcapacitor behave similarly to their conventional counterparts if $\gamma=1$. This asserts the aforesaid statement on the interpolate characteristic of these fractional-order memreactive devices. From (92) and (93), the following port equation of the fractional-order memreactance can be generally obtained:

$$
D^{1-\gamma}\left[u_{o}(t)\right]=X_{M}(t) u_{i}(t)
$$

where $\left\{u_{i}(t), u_{o}(t), X_{M}(t)\right\}$ can be either $\left\{i(t), \varphi(t), L_{M}(t)\right\}$ or $\left\{v(t), q(t), C_{M}(t)\right\}$ for the fractional-order meminductor and fractional-order memcapacitor, respectively.

Now, we will show that our mathematical model and its related results have been found to be applicable to the fractional-order memreactance with fractional-order $\mathrm{ki}$ netics as they determine $L_{M}(t)$ and $C_{M}(t)$. Thus, the effects of fractional-order kinetics on the fractional-order memreactance can be analyzed. By using (96) with appropriate sets of variables, $\varphi(t)$ and $v(t)$ due to arbitrary $i(t)$ can be, respectively, found as

$$
\begin{aligned}
& \varphi(t)=\varphi(0)+\frac{1}{\Gamma(1-\gamma)} \int_{0}^{t}(t-\tau)^{-\gamma} L_{M}(\tau) i(\tau) \mathrm{d} \tau, \\
& v(t)=\frac{C_{M}^{-1}(t)}{\Gamma(1+\gamma)} \frac{\mathrm{d}}{\mathrm{d} t} \int_{0}^{t}(t-\tau)^{\gamma} i(\tau) \mathrm{d} \tau,
\end{aligned}
$$

where $L_{M}(t)$ and $C_{M}(t)$ can be determined by using any of our equations of $\sqrt{L_{M}(t)}$ and $D_{M}(t)$ derived in Section 4 depending on the input, drift model, and assumption on $\alpha$. Here, we assume that $\gamma=0.5$ as this value is the midpoint between 0 and 1. Note that two extreme cases, i.e., $\gamma=0$ and $\gamma=1$, have already been considered in [7] and the previous sections of this work, respectively. This is because the fractional-order memreactance, respectively, becomes the memristor when $\gamma=0$ and memreactance when $\gamma=1$ as mentioned above. In addition, we let $i(t)=I_{m} \sin (\omega t+\theta)$. If we allow $\theta=\pi / 2 \mathrm{rad}, L_{\min }=1 \mathrm{mH}, L_{\max }=20 \mathrm{mH}$, and $L(0)=5 \mathrm{mH}$ similarly to the meminductor, the resulting asymptotic $\varphi(t)$ and $\varphi(t)-i(t)$ characteristics of the fractional-order meminductor can be simulated, as depicted in Figures 42-45 where $\varphi(0)=0 \mathrm{~Wb}$ and nonlinear dopant drift with $p=1$ have been assumed. From these figures, we have found that $\varphi(t)$ of the fractional-order meminductor employs a DC term which is positive if $I_{m}>0$, and vice versa. Moreover, such a DC term has been found to be directly proportional to $\alpha$. Note also that the fractional-order meminductor does not share the $2^{\text {nd }}$ basic fingerprint of the meminductor as its $\varphi(t)-i(t)$ hysteresis loop does not pinch but takes the elliptical shape which indicates that the fractional-order meminductor with the assumed $\gamma$ behaves like an AC resonator on the $\varphi(t)-i(t)$ plane, as can be seen from Figures 44 and 45 where the scaling has been applied to the vertical axis for visibility.

For the fractional-order memcapacitor on the contrary, we let $\theta=0 \mathrm{rad}, D_{\min }=0.1 \mathrm{MF}^{-1}, D_{\max }=0.1 \mathrm{GF}^{-1}$, and 


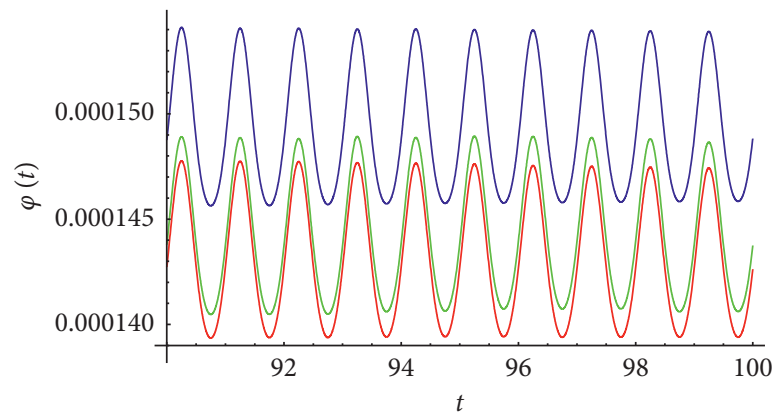

Figure 42: $\varphi(t)$ of the fractional-order meminductor with fractional kinetics: $\alpha=0.75$ (red), $\alpha=1$ (green), and $\alpha=1.25$ (blue) under the periodic input with $I_{m}>0$.

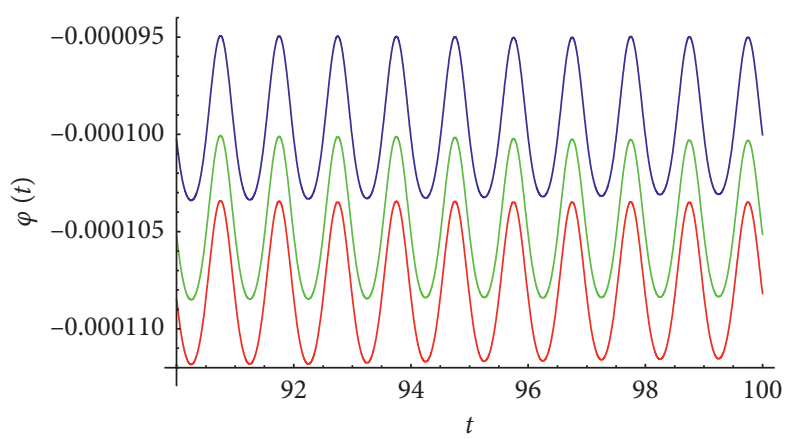

Figure 43: $\varphi(t)$ of the fractional-order meminductor with fractional kinetics: $\alpha=0.75$ (red), $\alpha=1$ (green), and $\alpha=1.25$ (blue) under the periodic input with $I_{m}<0$.

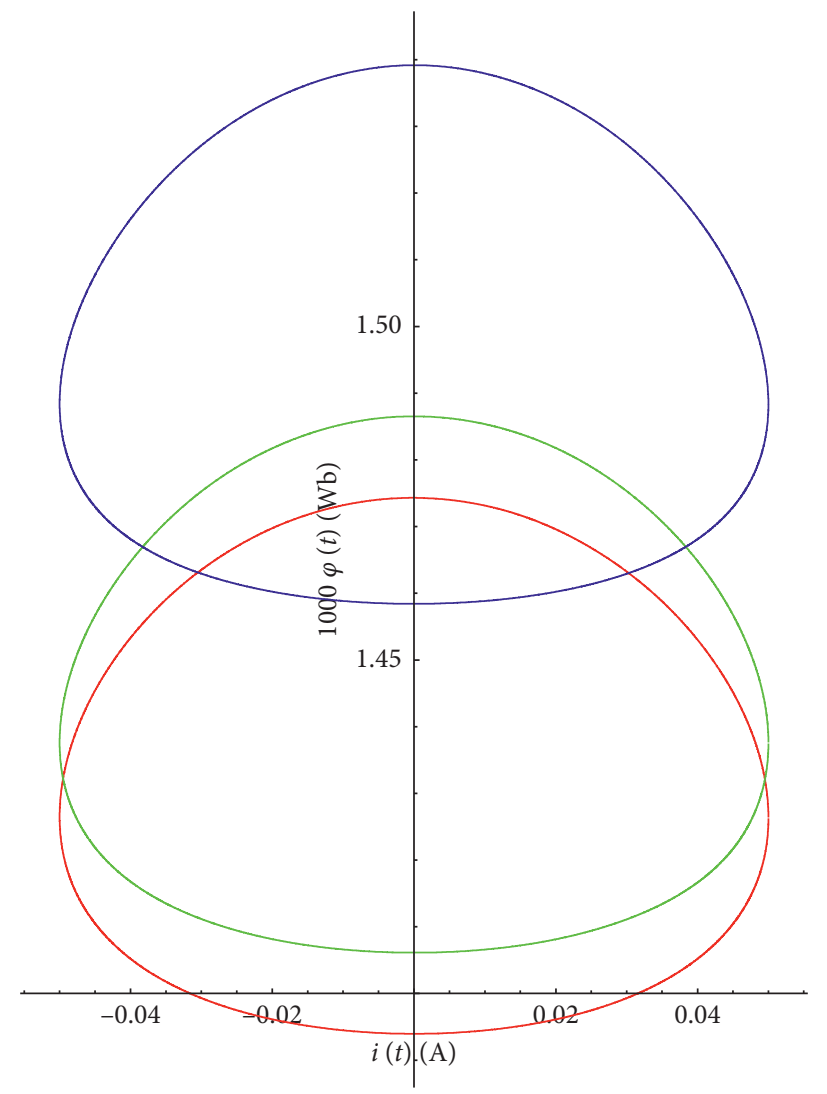

FIgURE 44: $\varphi(t)-i(t)$ of the fractional-order meminductor with fractional kinetics: $\alpha=0.75$ (red), $\alpha=1$ (green), and $\alpha=1.25$ (blue) under the periodic input with $I_{m}>0$. 


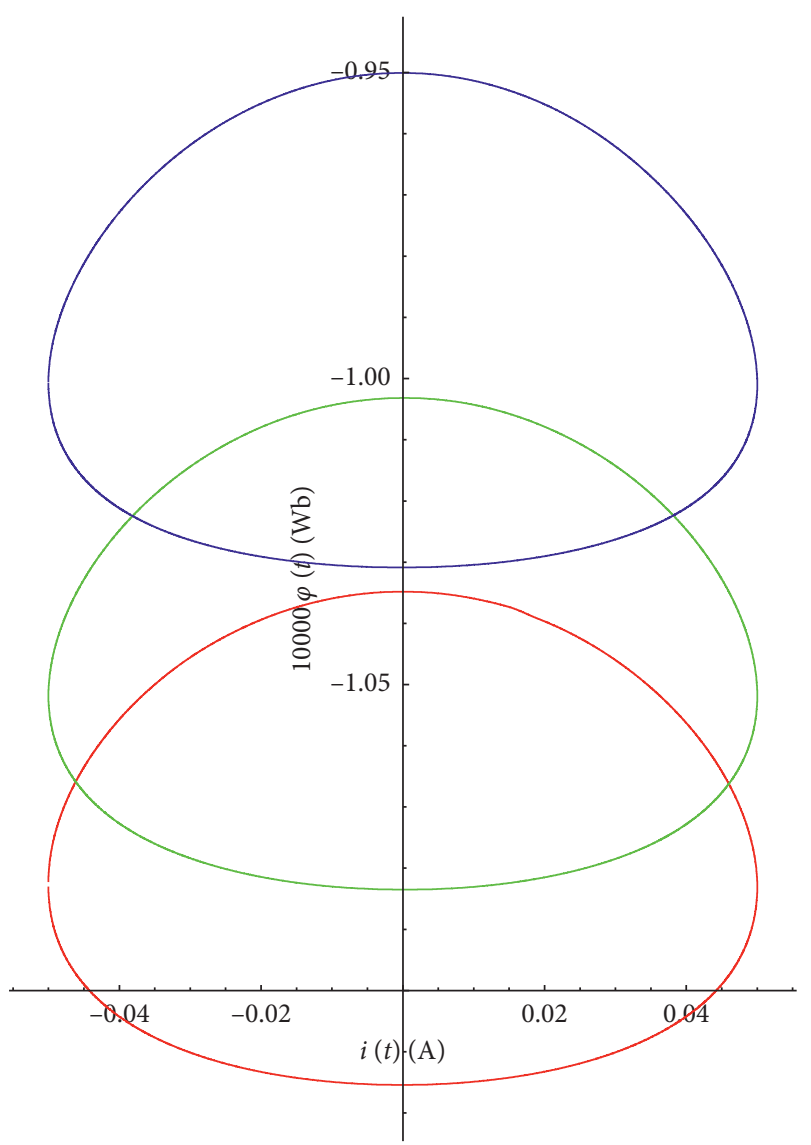

FIGURE 45: $\varphi(t)-i(\mathrm{t})$ of the fractional-order meminductor with fractional kinetics: $\alpha=0.75$ (red), $\alpha=1$ (green), and $\alpha=1.25$ (blue) under the periodic input with $I_{m}<0$.

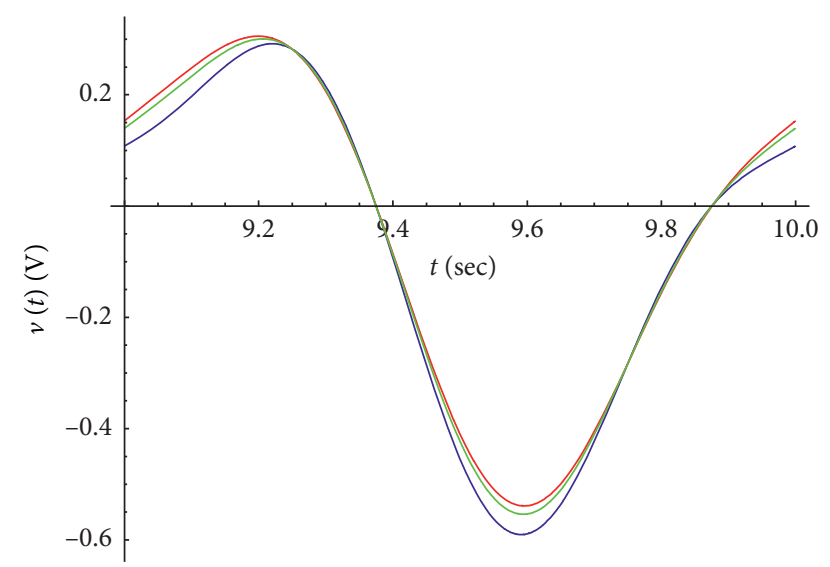

FIgURE 46: $v(t)$ of the fractional-order memcapacitor with fractional kinetics: $\alpha=0.75$ (red), $\alpha=1$ (green), and $\alpha=1.25$ (blue) under the periodic input with $I_{m}>0$.

$D(0)=0.01 \mathrm{GF}^{-1}$ as well as for the memcapacitor. The simulated asymptotic $v(t)$ and $v(t)-q(t)$ characteristics of the fractional order can be depicted in Figures 46-49 where the nonlinear dopant drift with $p=1$ has been assumed. From Figures 46 and 47, we have found that the distortion of $v(t)$ is directly proportional to $\alpha$. From Figures 48 and 49 where the vertical axis has been scaled for visibility, it can be seen

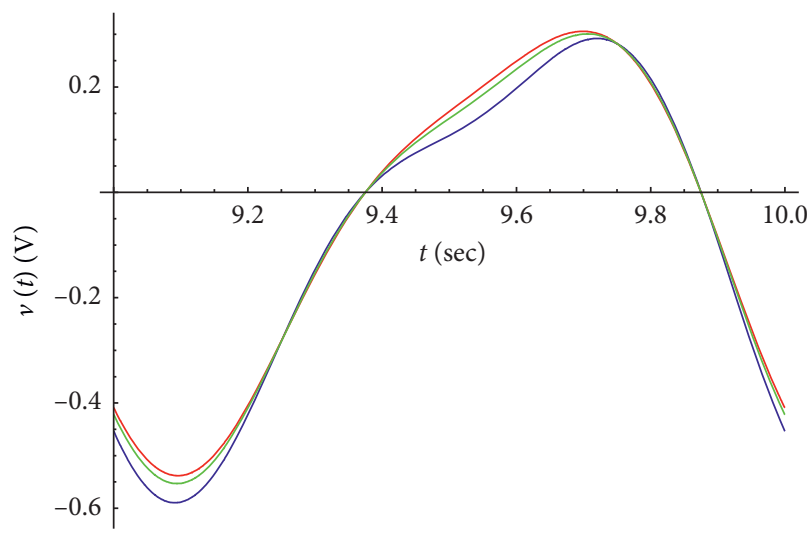

FIGURE 47: $v(t)$ of the fractional-order memcapacitor with fractional kinetics: $\alpha=0.75$ (red), $\alpha=1$ (green), and $\alpha=1.25$ (blue) under the periodic input with $I_{m}>0$.

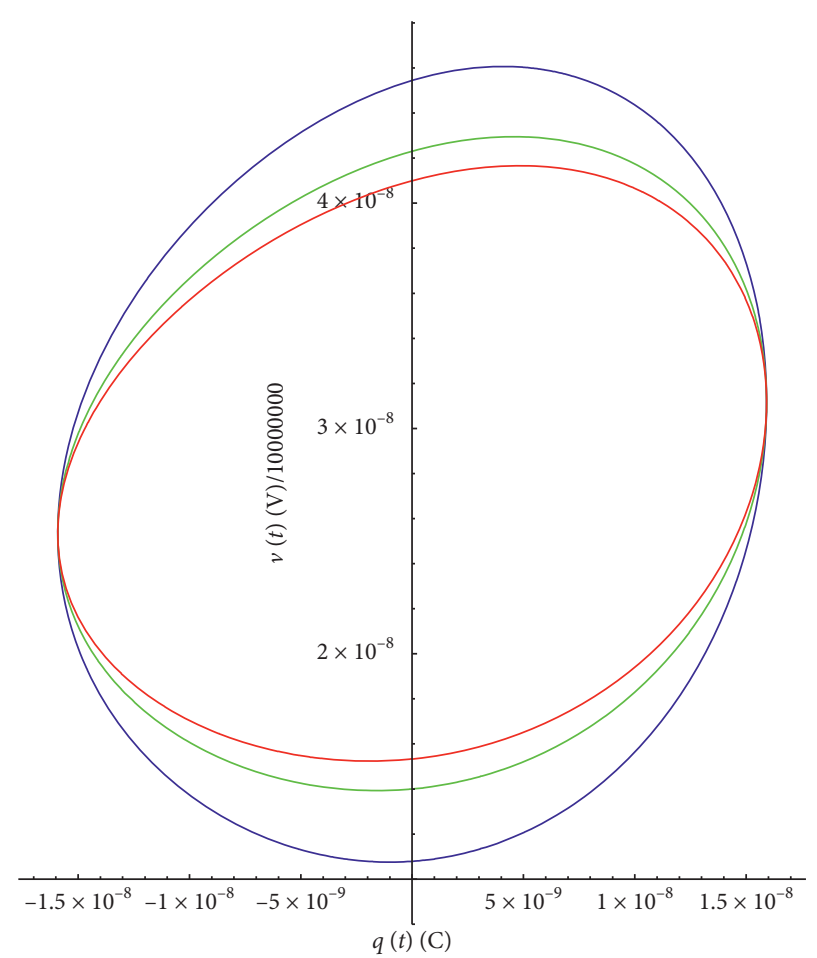

FIgURE 48: $v(t)-q(t)$ of the fractional-order memcapacitor with fractional kinetics: $\alpha=0.75$ (red), $\alpha=1$ (green), and $\alpha=1.25$ (blue) under the periodic input with $I_{m}>0$.

that the area of the hysteresis loop of the fractional-order memcapacitor is directly proportional to $\alpha$; thus, less linearity can be obtained by using the fractional-order memcapacitor with higher $\alpha$. This is in contrast to the behavior of the memcapacitor. Similarly to the fractional-order meminductor, the fractional-order memcapacitor does not share the $2^{\text {nd }}$ basic fingerprint of the memcapacitor as its $v(t)-q(t)$ hysteresis loop does not pinch but takes the elliptical shape which implies that the fractional-order memcapacitor with the assumed $\gamma$ behaves like an AC resonator on the $v(t)-q(t)$ plane. 


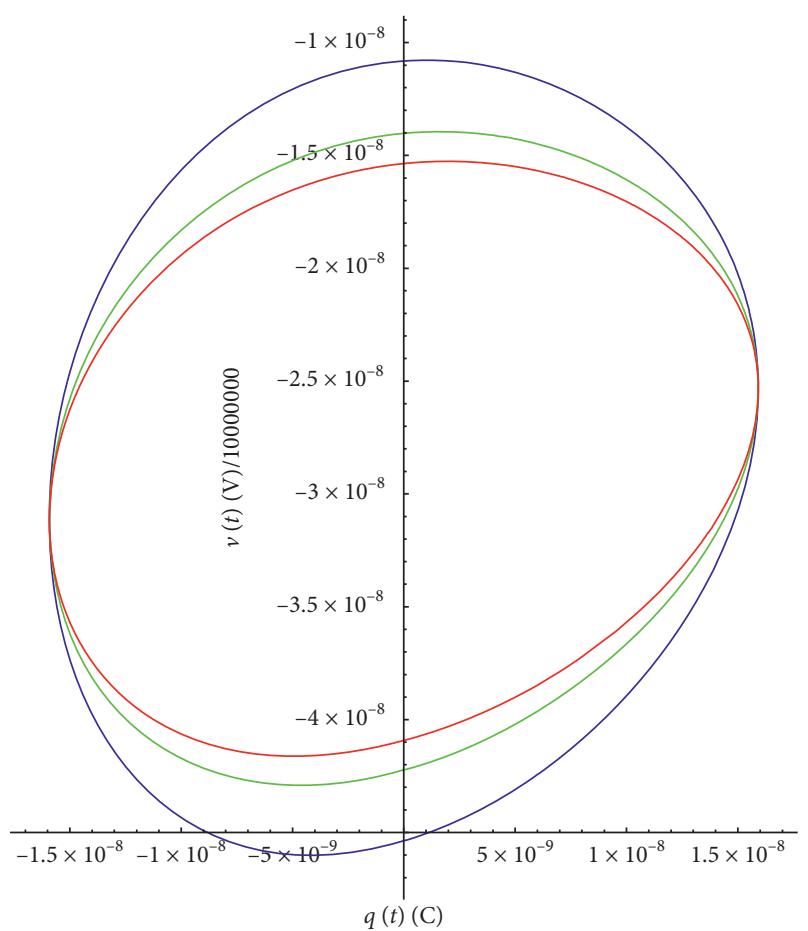

FIGURE 49: $v(t)-q(t)$ of the fractional-order memcapacitor with fractional kinetics: $\alpha=0.75$ (red), $\alpha=1$ (green), and $\alpha=1.25$ (blue) under the periodic input with $I_{m}<0$.

\section{Conclusion}

We analyze the memreactance with fractional-order kinetic transport in this work by means of fractional calculus. Novel fractional-order kinetics involved analytical expressions of memreactance in general; meminductances, memcapacitances, and related parameters due to both DC and periodic input waveforms including those with the nonlinear dopant drift which are rather complicated have been derived. Moreover, the behavioral analysis has been thoroughly performed by using the derived equations and numerical simulations. As a result, the effects of fractional-order kinetics have been thoroughly explored where both linear and nonlinear dopant drift scenarios have been considered. In summary, it can be seen from the simulation results that the fractional-order kinetic transport significantly affects the behavior of memreactance due to both DC and periodic inputs, e.g., saturation time and nonlinearity, where the memreactance with the nonlinear dopant drift is more robust to the effects of fractional-order kinetics as stated above. For performing the numerical simulations in this work, higher computational effort compared to those of the previous works on the conventional memreactance with integer kinetics $[5,33,34]$ is required due to more complexity of our equations, yet higher real memreactance modelling accuracy can be obtained in return because the real electronic device employs fractional-order kinetics in practice [22] and so does the memreactance. Moreover, the memreactance emulation by using the memristor with fractional-order kinetics, the effect of fractional-order kinetics on the memreactance-based circuits, and the application of our results to the fractional-order memreactance have also been presented. The results of this work have been found to be beneficial to those memelementinvolved research areas.

\section{Data Availability}

The simulated data used to support the findings of this study are included within this article.

\section{Conflicts of Interest}

The author declares that there are no conflicts of interest regarding the publication of this article.

\section{Acknowledgments}

The author would like to acknowledge Mahidol University, Thailand, for the online database service which is our primary information resource.

\section{References}

[1] L. Chua, "Memristor-the missing circuit element," IEEE Transactions on Circuit Theory, vol. CT-18, no. 5, pp. 507-519, 1971.

[2] M. Di Ventra, Y. V. Pershin, and L. O. Chua, "Circuit elements with memory: memristors, memcapacitors, and meminductors," Proceedings of the IEEE, vol. 97, no. 10, pp. 1717-1724, 2009.

[3] M. E. Fouda and A. G. Radwan, "On the fractional-order memristor model," Journal of Fractional Calculus and Applications, vol. 4, no. 1, pp. 1-7, 2013.

[4] M. E. Fouda and A. G. Radwan, "Fractional-order memristor response under dc and periodic signals," Circuits, Systems, and Signal Processing, vol. 34, no. 3, pp. 961-970, 2015.

[5] A. G. Radwan and M. E. Fouda, On the Mathematical Modeling of Memristor, Memcapacitor, and Meminductor, Vol. 26, Springer, Berlin, Germany, 2015.

[6] G. Si, L. Diao, J. Zhu, Y. Lei, O. Babajide, and Y. Zhang, "Modeling and character analyzing of current-controlled memristors with fractional kinetic transport," Communications in Nonlinear Science and Numerical Simulation, vol. 48, pp. 224-235, 2017.

[7] R. Banchuin, "On the memristances, parameters, and analysis of the fractional order memristor," Active and Passive Electronic Components, vol. 2018, Article ID 3408480, 14 pages, 2018.

[8] I. Podlubny, Fractional Differential Equations, Academic Press, New York, USA, 1999.

[9] M. Caputo and M. Fabrizio, "A new definition of fractional derivative without singular kernel," Progress in Fractional Differentiation and Applications, vol. 1, no. 2, pp. 73-85, 2015.

[10] A. Atangana and D. Baleanu, "New fractional derivatives with nonlocal and non-singular kernel: theory and application to heat transfer model," Thermal Science, vol. 20, no. 2, pp. 763-769, 2016.

[11] I. S. Jesus, J. A. Tenreiro Machado, and J. Boaventure Cunha, "Fractional electrical impedances in botanical elements," Journal of Vibration and Control, vol. 14, no. 9-10, pp. 1389-1402, 2008.

[12] C. Tang, F. You, G. Cheng, D. Gao, F. Fu, and X. Dong, "Modeling the frequency dependence of the electrical 
properties of the live human skull," Physiological Measurement, vol. 30, no. 12, pp. 1293-1301, 2009.

[13] A. Charef, "Analogue realisation of fractional-order integrator, differentiator and fractional PI $\lambda \mathrm{D} \mu$ controller," IEE Proceedings-Control Theory and Applications, vol. 153, no. 6, pp. 714-720, 2006.

[14] B. M. Vinagre and V. Feliu, "Optimal fractional controllers for rational order systems: a special case of the Wiener-Hopf spectral factorization method," IEEE Transactions on Automatic Control, vol. 52, no. 12, pp. 2385-2389, 2007.

[15] R. Matusu, "Application of fractional order calculus to control theory," International Journal of Mathematical Models and Methods in Applied Sciences, vol. 5, pp. 1162-1169, 2011.

[16] L. Dork, J. Terpk, I. Petr, and F. Dorkov, "Electronic realization of the fractional-order systems," Acta Montanistica Slovaca, vol. 12, pp. 231-237, 2007.

[17] B. T. Krishna, K. V. V. S. Reddy, and S. Santha Kumari, “Time domain response calculations of fractance device of order 1/ 2," The Journal of Active and Passive Electronic Devices, vol. 3, pp. 355-367, 2008.

[18] A. G. Radwan and A. S. Elwakil, "An expression for the voltage response of a current-excited fractance device based on fractional-order trigonometric identities," International Journal of Circuit Theory and Applications, vol. 40, no. 5, pp. 533-538, 2012.

[19] B. Baykant Alagoz and H. Alisoy, "Estimation of reduced order equivalent circuit model parameters of batteries from noisy current and voltage measurements," Balkan Journal of Electrical and Computer Engineering, vol. 6, no. 4, pp. 224231, 2018.

[20] B. B. Alagoz, G. Alisoy, S. Alagoz, and H. Alisoy, "A note on applications of time-domain solution of Cole permittivity models," Optik, vol. 139, pp. 272-282, 2017.

[21] B. B. Alagoz, A. Tepljakov, A. Ates, E. Petlenkov, and C. Yeroglu, "Time-domain identification of one noninteger order plus time delay models from step response measurements," International Journal of Modeling, Simulation, and Scientific Computing, vol. 10, no. 1, Article ID 1941011, 2019.

[22] J. F. Gómez-Aguilar, A. Atangana, and V. F. Morales-Delgado, "Electrical circuits RC, LC, and RL described by Atangana-Baleanu fractional derivatives," International Journal of Circuit Theory and Applications, vol. 45, no. 11, pp. 1514-1533, 2017.

[23] J. Gómez-Aguilar, V. Morales-Delgado, M. Taneco-Hernández, D. Baleanu, R. Escobar-Jiménez, and M. Al Qurashi, "Analytical solutions of the electrical RLC circuit via Liouville-Caputo operators with local and non-local kernels," Entropy, vol. 18, no. 8, p. 402, 2016.

[24] J. F. Gómez Aguilar and D Baleanu, "Solutions of the telegraph equations using a fractional calculus approach," Proceedings of the Romanian Academy-Series A, vol. 15, pp. 27-34.

[25] J. F. Gómez-Aguilar, H. Yépez-Martínez, R. F. EscobarJiménez, C. M. Astorga-Zaragoza, and J. Reyes-Reyes, “Analytical and numerical solutions of electrical circuits described by fractional derivatives," Applied Mathematical Modelling, vol. 40, no. 21-22, pp. 9079-9094, 2016.

[26] J. F. Gómez-Aguilar and B. Dumitru, "Fractional transmission line with losses," Zeitschrift für Naturforschung A, vol. 69, no. 10-11, pp. 539-546, 2014.

[27] F. Gómez, J. Rosales, and M. Guía, "RLC electrical circuit of non-integer order," Open Physics, vol. 11, no. 10, pp. 1361-1365, 2013.
[28] J. I. Hidalgo-Reyes, J. F. Gómez-Aguilar, R. F. EscobarJiménez, V. M. Alvarado-Martínez, and M. G. López-López, "Classical and fractional-order modeling of equivalent electrical circuits for supercapacitors and batteries, energy management strategies for hybrid systems and methods for the state of charge estimation: a state of the art review," Microelectronics Journal, vol. 85, pp. 109-128, 2019.

[29] V. F. Morales-Delgado, J. F. Gómez-Aguilar, M. A. TanecoHernández, and R. F. Escobar-Jiménez, "Fractional operator without singular kernel: applications to linear electrical circuits," International Journal of Circuit Theory and Applications, vol. 46, no. 12, pp. 2394-2419, 2018.

[30] "Equivalent circuits applied in electrochemical impedance spectroscopy and fractional derivatives with and without singular kernel," Advances in Mathematical Physics, vol. 2016, Article ID 9720181, 16 pages, 2016.

[31] M. S. Abdelouahab, R. Lozi, and L. Chua, "Memfractance: a mathematical paradigm for circuit elements with memory," International Journal of Bifurcation and Chaos, vol. 24, no. 9, Article ID 1430023, 2014.

[32] L. Fu and H. Yang, "An application of $(3+1)$-dimensional time-space fractional ZK model to analyze the complex dust acoustic waves," Complexity, vol. 2019, Article ID 2806724, 15 pages, 2019.

[33] R. Tian, L. Fu, Y. Ren, and H. Yang, "(3+1)-Dimensional timefractional modified Burgers equation for dust ion-acoustic waves as well as its exact and numerical solutions," Mathematical Methods in the Applied Sciences, vol. 1-20, 2019.

[34] M. E. Fouda and A. G. Radwan, "Meminductor response under periodic current excitations," Circuits, Systems, and Signal Processing, vol. 33, no. 5, pp. 1573-1583, 2014.

[35] M. E. Fouda and A. G. Radwan, "Memcapacitor response under step and sinusoidal voltage excitations," Microelectronics Journal, vol. 45, no. 11, pp. 1372-1379, 2014.

[36] G. Scarpa, J. A. Russer, P. Lugli, and P. Russer, "Quantum dynamics of circuits with memory capacitors and inductors," in Proceedings of the 2012 International Conference on Electromagnetics in Advanced Applications, pp. 614-617, IEEE, Cape Town, South Africa, September 2012.

[37] G. Y. Wang, P. P. Jin, X. W. Wang, Y. R. Shen, F. Yuan, and $\mathrm{X}$. Y. Wang, "A flux-controlled model of meminductor and its application in chaotic oscillator," Chinese Physics B, vol. 25, no. 9, Article ID 090502, 2016.

[38] C. Li, C. Li, T. Huang, and H. Wang, "Synaptic memcapacitor bridge synapses,” Neurocomputing, vol. 122, pp. 370-374, 2013.

[39] M. E. Fouda and A. G. Radwan, "On the mathematical modeling of memcapacitor bridge synapses," in Proceedings of the 2014 26th International Conference on Microelectronics (ICM), pp. 172-175, IEEE, Doha, Qatar, December 2014.

[40] D. Biolek, Z. Biolek, and V. Biolková, "PSPICE modeling of meminductor," Analog Integrated Circuits and Signal Processing, vol. 66, no. 1, pp. 129-137, 2011.

[41] D. Biolek, Z. Biolek, and V. Biolkova, "SPICE modelling of memcapacitor," Electronics Letters, vol. 46, no. 7, pp. 520-522, 2010.

[42] W. H. Beyer, CRC Handbook of Mathematical Sciences, CRC Press, Boca Raton, FL, USA, 1987.

[43] B. Dwork, Generalized Hypergeometric Functions, Clarendon Press, Oxford, UK, 1990.

[44] M. Abramowitz and I. A. Stegun, "Sine and cosine integrals," in Section 5.2 in Handbook of Mathematical Functions with Formulas, Graphs, and Mathematical Tables, pp. 231-233, Dover, New York, USA, 9th edition, 1972. 
[45] A. A. Kilbas, H. M. Srivastava, and J. J. Trujillo, Theory andApplications of Fractional Differential Equations, vol. 204, Elsevier, Amsterdam, Netherlands, 2006.

[46] A. D. Polyanin and A. V. Manzhirov, Handbook of Mathematics for Engineers and Scientists, Chapman and Hall/CRC, London, UK, 2006.

[47] D. Biolek and V. Biolkova, "Mutator for transforming memristor into memcapacitor," Electronics Letters, vol. 46, no. 21, pp. 1428-1429, 2010. 


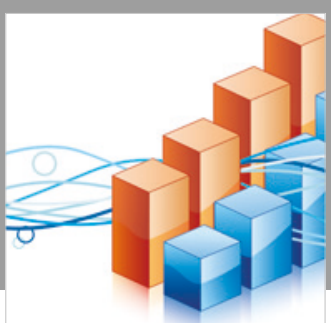

Advances in

Operations Research

\section{-n-m}
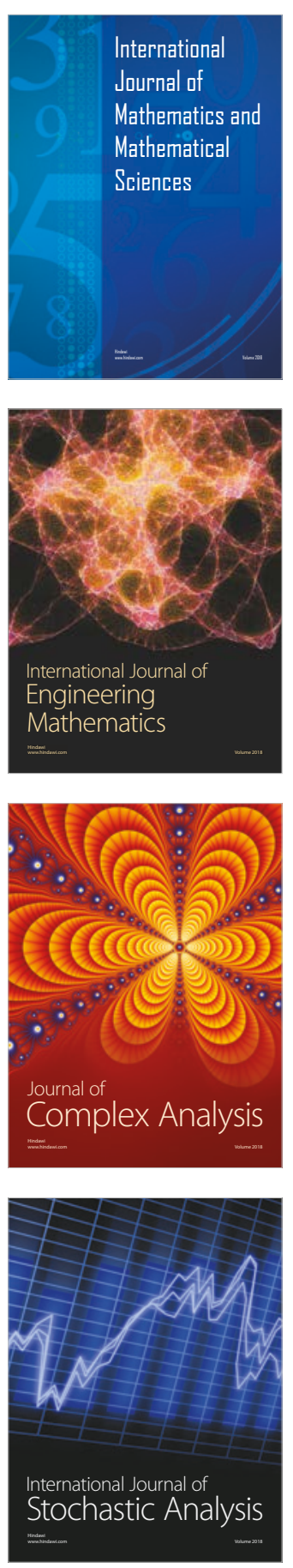
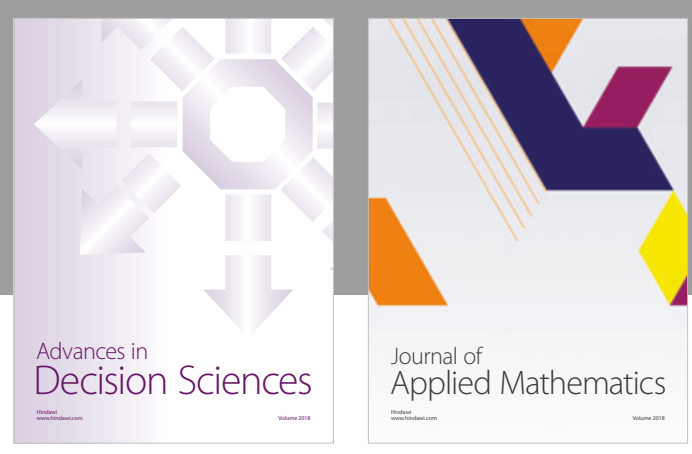

Journal of

Applied Mathematics
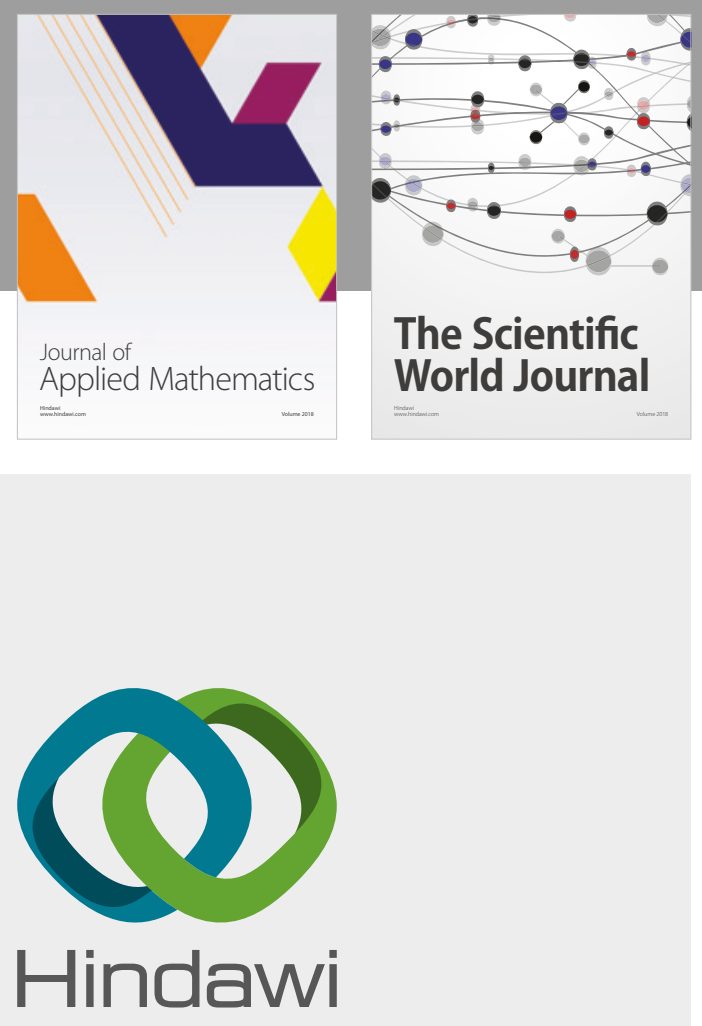

Submit your manuscripts at

www.hindawi.com

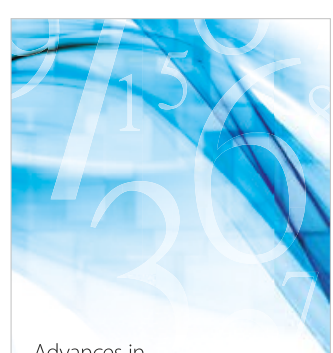

Advances in
Numerical Analysis
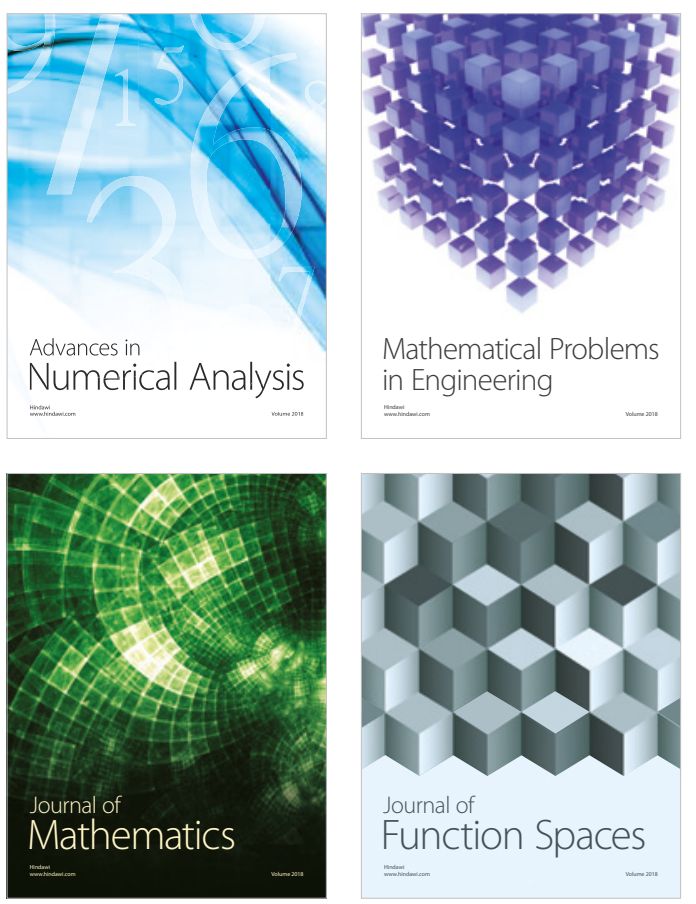

Mathematical Problems in Engineering

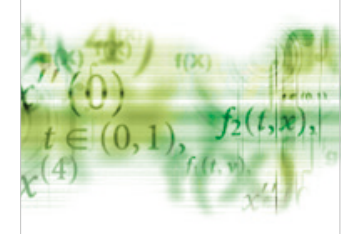

International Journal of

Differential Equations

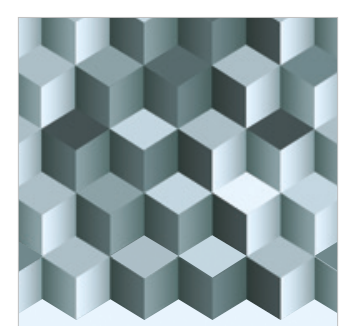

Journal of

Function Spaces

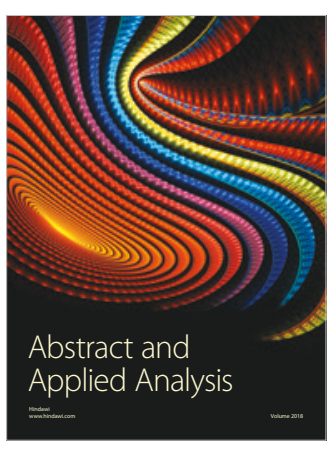

The Scientific

World Journal

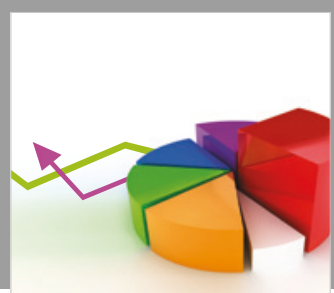

Journal of

Probability and Statistics
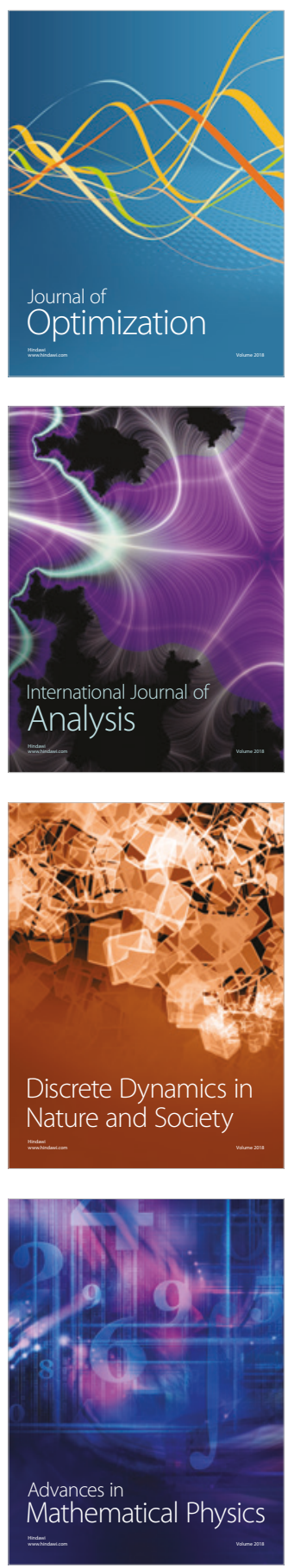